\title{
Turbulent boundary layers over permeable walls: scaling and near-wall structure
}

\author{
C. Manes $\nmid+$, D. Poggi and L. Ridolfi \\ Politecnico di Torino, Dipartimento di Idraulica, Trasporti ed Infrastrutture Civili (DITIC), \\ Corso Duca degli Abruzzi, 10129 Torino, Italy
}

(Received 22 February 2011; revised 25 July 2011; accepted 30 July 2011;

first published online 10 October 2011)

This paper presents an experimental study devoted to investigating the effects of permeability on wall turbulence. Velocity measurements were performed by means of laser Doppler anemometry in open channel flows over walls characterized by a wide range of permeability. Previous studies proposed that the von Kármán coefficient associated with mean velocity profiles over permeable walls is significantly lower than the standard values reported for flows over smooth and rough walls. Furthermore, it was observed that turbulent flows over permeable walls do not fully respect the widely accepted paradigm of outer-layer similarity. Our data suggest that both anomalies can be explained as an effect of poor inner-outer scale separation if the depth of shear penetration within the permeable wall is considered as the representative length scale of the inner layer. We observed that with increasing permeability, the near-wall structure progressively evolves towards a more organized state until it reaches the condition of a perturbed mixing layer where the shear instability of the inflectional mean velocity profile dictates the scale of the dominant eddies. In our experiments such shear instability eddies were detected only over the wall with the highest permeability. In contrast attached eddies were present over all the other wall conditions. On the basis of these findings, we argue that the near-wall structure of turbulent flows over permeable walls is regulated by a competing mechanism between attached and shear instability eddies. We also argue that the ratio between the shear penetration depth and the boundary layer thickness quantifies the ratio between such eddy scales and, therefore, can be used as a diagnostic parameter to assess which eddy structure dominates the near-wall region for different wall permeability and flow conditions.

Key words: geophysical and geological flows, shear layer turbulence, turbulent boundary layers

\section{Introduction}

The study of turbulent flows over permeable walls is of practical and theoretical interest. The practical interest is associated with the need to predict scalar transport processes in many geophysical and industrial flows which are often characterized by

$\dagger$ Email address for correspondence: costantino.manes@polito.it

$\ddagger$ Present address: Politecnico di Torino, Dipartimento di Idraulica, Trasporti ed Infrastrutture Civili (DITIC), Corso Duca degli Abruzzi, Torino, Italy. 
permeable walls (gravel bed rivers, atmospheric flows over vegetation, heat-exchangers, coastal protection structures, etc.). From a theoretical point of view, turbulent flows over permeable walls represent an attractive opportunity to investigate how the relaxation of the wall blocking condition, promoted by wall permeability, influences the dynamics of wall turbulence. The incentive to study this problem comes from the widespread idea that the wall blocking condition plays an important role in the dynamics of coherent structures typically observed in turbulent boundary layers over smooth or rough walls (Krogstad, Antonia \& Browne 1992; Hunt \& Morrison 2000).

Investigating the effects of wall permeability on turbulent flows is not an easy task. The difficulty lies in the nature of the walls, which are inherently permeable and rough; therefore, in numerical or physical experiments it becomes difficult to differentiate between the effects of permeability and roughness. In the literature, this problem has been overcome by following two approaches. The first, particularly used in the study of flows over granular beds, involves a comparative analysis between flow statistics measured over rough impermeable and rough permeable walls characterized by the same surface roughness texture (Zagni \& Smith 1976; Manes et al. 2009). For example Manes et al. (2009) present a comparison between statistics of velocities measured in open channel flows over an impermeable wall made of one layer of beads and a permeable wall made of five of such layers. The rationale behind this approach lies in the concept that (i) a wall is considered as permeable (impermeable) if its thickness is significantly larger (comparable or smaller) than the characteristic size of its constitutive components; (ii) the comparison between flow statistics measured over the two beds highlights the effects of wall permeability since the surface roughness is kept the same. Results from the studies that applied this approach indicate that wall permeability significantly influences flow resistance. It was observed that for a given relative roughness (i.e. ratio between flow thickness and equivalent grain size) friction factors increase with increasing Reynolds number $(R e)$ without reaching a hydraulically rough plateau, which is typical of impermeable rough walls at similar Re. Manes et al. (2011) argue that the increase in friction factors is associated with a progressive shear penetration within the permeable wall and/or with a persistent viscous drag exerted by sheltered grains.

The second approach has been proposed by Hahn, Je \& Choi (2002) and then refined by Breugem, Boersma \& Uittenbogaard (2006). Breugem et al. (2006) suggest that a permeable wall is characterized by three length scales, namely the square root of permeability $\sqrt{K}$, the wall thickness $H$, and the characteristic size of the 'roughness' elements $d_{p}$ composing the solid matrix of the wall. The effect of permeability on the flow is isolated if (i) the wall thickness is larger than the turbulence penetration depth; (ii) the roughness Reynolds number $R e_{d}=d_{p} u_{*} / v$ is sufficiently small (e.g. $\left.R e_{d} \ll 70\right)$ and (iii) the permeability Reynolds number $R e_{K}=\sqrt{K} u_{*} / v$ is sufficiently high, i.e. $R e_{K}>1$ (in the above formulas $u_{*}$ is the friction velocity and $v$ is the kinematic viscosity of the fluid). In line with this argument, Breugem et al. (2006) performed direct numerical simulations (DNS) of channel flows over permeable walls characterized by small cubical elements packed in a very open cubic pattern. The results showed that permeability alters significantly the structure and dynamics of wall turbulence. The more permeable the wall is, the less evident the high- and low-speed streaks associated with quasi-streamwise vortices typical of impermeable smooth walls become. This result was explained partly by the reduction in shear due to the relaxation of the no-slip condition and partly by the enhanced turbulent transport of fluid across the wall surface, which in turn is associated with the weakening 
of the wall blocking effect. The structure of turbulence in the proximity of the permeable wall was observed to be dominated by large vortical structures associated with Kelvin-Helmoltz $(\mathrm{KH})$ instabilities induced by the inflection of the mean velocity profile at the wall surface.

In the simulations performed by Breugem et al. (2006) the log law of the wall could be fitted to the mean velocity profiles over the permeable walls. The resulting von Kármán coefficients $\kappa$ were significantly smaller than the standard values reported for smooth or rough walls. Similar results were also obtained by Suga et al. (2010). Breugem et al. (2006) argue that this anomaly is either due to the alteration of the flow structure induced by wall permeability or is an effect of the low Reynolds numbers at which simulations were carried out. Therefore, they recommended carrying out further studies at higher Reynolds numbers. These authors also noticed that Townsend's hypothesis of outer similarity (Townsend 1976) was respected for some statistical quantities and not for others. In particular the root mean square (r.m.s.) of the vertical velocity fluctuations did not exhibit similarity. As for the von Kármán coefficient, they argued that such a deviation could be either another shortcoming of the low Reynolds numbers of the simulations, or an effect induced by the weakening of the wall blocking condition. According to the latter hypothesis, when the wall blocking condition is relaxed, strong sweeps and/or ejections can be transported across the entire boundary layer depth and hence allow significant communication between the inner and the outer layer. Therefore, the inner flow, which is governed by the wall characteristics, directly influences the outer layer, thus preventing similarity.

Besides the work of Breugem et al. (2006), the wealth of numerical and experimental studies dedicated to turbulent flows over vegetation canopies provide interesting results that can be exploited for the study of turbulent flows over permeable walls in a more general sense. However, the results from the canopy flow literature have to be taken with care, because most of the numerical or physical experiments in this research area were not designed to investigate the effects of permeability on wall turbulence exclusively and the conditions listed by Breugem et al. (2006) to do so, were not fully met. In particular, the penetration depth of turbulence is usually comparable with the canopy height (Finnigan 2000), which therefore remains a relevant scale for the flow. Despite these limitations, the literature on canopy flows remains an important reference and it is briefly summarized in the following.

It is now widely accepted that, if the canopy is dense enough, the near-wall flow behaves as a perturbed mixing layer (Raupach, Finnigan \& Brunet 1996; Ghisalberti \& Nepf 2002; Poggi et al. 2004). The inflection of the mean velocity profile at the canopy top is dynamically active and represents the primary cause of the formation of coherent structures, characterized by energetic sweeps and less energetic ejections (Finnigan 2000; Finnigan, Shaw \& Patton 2009). Finnigan et al. (2009) present a model for the dynamics of such coherent structures where the hydrodynamic instability at the canopy top, associated with the inflection in mean shear, is responsible for a strong scale selection which makes the flow at the canopy top more coherent than over other rough surfaces. The dominance of sweeps over ejections is justified by the permeable nature of the canopy which allows the generation of significant downward deflections. By means of conditional sampling techniques, Finnigan et al. (2009) observed that sweeps and ejections are associated with head-down and head-up hairpin vortices respectively. It was also observed that, in analogy with other rough surfaces, at a certain distance from the canopy top, ejections start to dominate over sweeps. This crossover is explained as a result of two counteracting processes: away from the wall (i.e. at heights much bigger than the canopy height) downwards motions of scales 
comparable to the vertical height $z$ are blocked by the wall (Hunt \& Morrison 2000) so the probability of upward deflections of hairpin vortices, and therefore of ejections, exceeds that of sweeps (i.e. downward deflections). In contrast, near the wall, the porous canopy allows downwards deflections as much as for upward deflections, but the former are also strained and amplified more than the latter due to the presence of mean shear. This guarantees that sweeps dominate near the canopy top. Finnigan et al. (2009) suggest that the crossover between sweep- and ejection-dominated regions could be a function of the canopy structure and, in particular, of the vorticity thickness associated with the inflectional mean velocity profile at the canopy top.

In the present study we investigate the effects of permeability on the dynamics of wall turbulence. We follow the approach suggested by Breugem et al. (2006) as it allows us to investigate the effects of permeability exclusively without the need of comparative analysis between flows over walls sharing the same roughness texture. To this end, we carried out a series of experiments where velocity fluctuations were measured by means of a laser Doppler anemometer (LDA) in open channel flows over three permeable beds covering a range of wall permeability spanning two orders of magnitude. As will be shown in detail, the paper reports how the scaling and the structure of turbulent flows over permeable walls evolve when wall permeability varies between two limiting conditions: (i) an impermeable smooth wall; (ii) a very permeable wall resembling a canopy which imposes a well-defined mixing layer in the near-wall region. The first represents the natural limiting condition of a wall with zero permeability whereas the second was chosen as it includes flow processes that are very relevant for geophysical applications. The transition between the two limiting conditions will be assessed in terms of scaling of the mean velocity profile, the magnitude of turbulence intensities (i.e. r.m.s. of velocity fluctuations), and characterization of the near-wall structure, which will be investigated by means of quadrant and spectral analysis. Some of the experiments were carried out with the highest Reynolds numbers ever attained for flows over permeable walls. This allowed us to test the results of Breugem et al. (2006) on the von Kármán coefficient and the outer-layer similarity. Furthermore, we investigate what are the dominant eddies responsible for turbulent kinetic energy (TKE) production in the near-wall region of turbulent flows over permeable walls and, in particular, what are the flow and wall conditions that allow the near-wall structure to reach the limiting case of a perturbed mixing layer.

The paper is organized as following. In the following section we describe the experimental equipment and procedure. Section 3 is devoted to the presentation of the results, where we discuss the validity of the log law of the wall, the validity of the outer-layer similarity hypothesis and the turbulence structure above permeable walls. Finally, in $\S 4$ we present a summary and a discussion of the main results.

\section{Experimental methodology}

\subsection{Materials}

The experiments involve turbulence measurements in open channel flows over three permeable walls and a smooth wall. The smooth wall was composed of stainless steel and represents the first limiting condition of a perfectly impermeable wall. The permeable walls were composed of three types of open-cell polyurethane foam each of them characterized by a different permeability (see figure 1). As will be discussed in the results section, the wall with the highest permeability represents the second limiting condition since it allows the development of a flow resembling a 


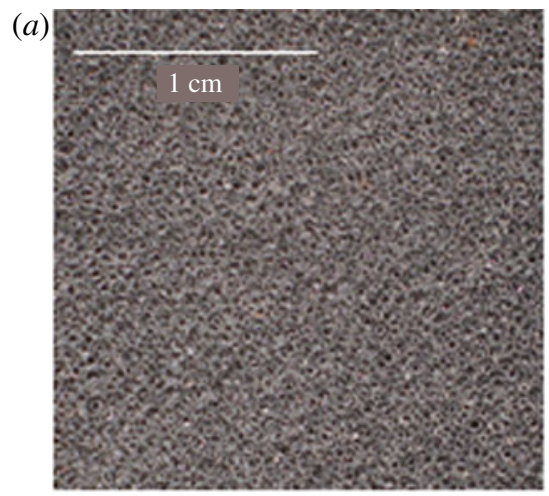

(b)

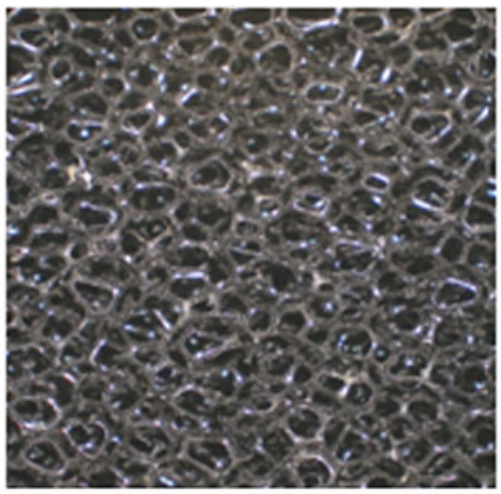

$(c)$
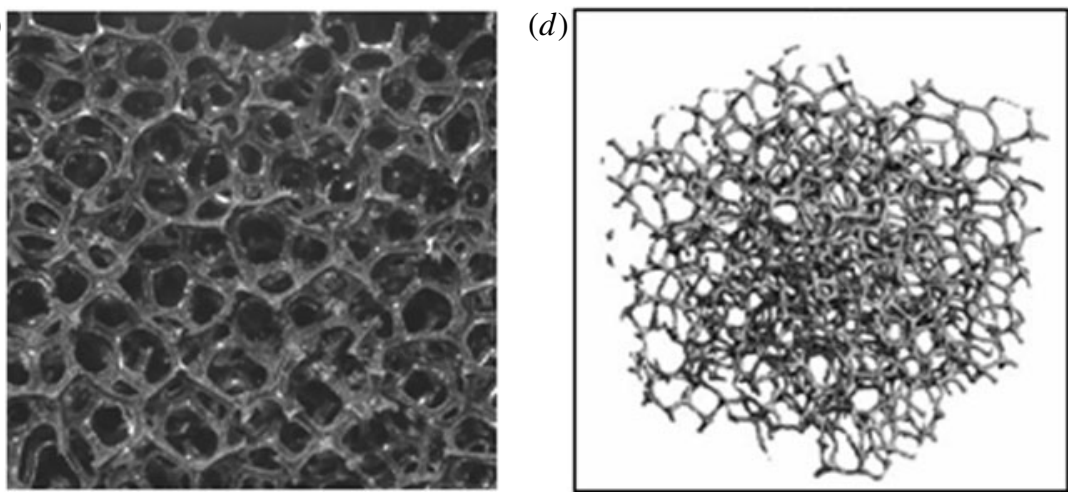

FIGURE 1. Foam samples: (a) 60 pores per inch (ppi); (b) 30 ppi; (c) 10 ppi; $(d)$ sample of the digitized X-ray tomography used to obtain the foam characteristics reported in table 1.

perturbed mixing layer. The permeability and the relevant geometrical properties of each foam type are the same as in Clifton et al. (2008) and are reported in table 1. The permeability of each wall was obtained by means of a standard 'Darcy-type' experiment. This was performed by placing a sample of porous media within a closed duct where an air flow rate was imposed and the associated pressure drop was measured (Clifton et al. 2008). With respect to Clifton et al. (2008) we also report the mean thickness of the filaments composing the foam structure since we argue that this length scale is associated with the 'roughness' dimension $d_{p}$ of each permeable wall. The specific surface area (SSA), the mean pore size and the filament thickness of the foam reported in table 1 were estimated from X-ray tomography (see figure $1 d$ ) of foam samples following the procedure outlined in Schneebeli \& Sokratov (2004). The spatial resolution of the tomography was $10 \mu \mathrm{m}, 20 \mu \mathrm{m}$ and $36 \mu \mathrm{m}$ for foam type $60 \mathrm{ppi}, 30 \mathrm{ppi}$ and $10 \mathrm{ppi}$ respectively. The SSA was computed as the total surface of the filaments divided by the total solid volume, and the mean pore size $\left(l_{\text {pore }}\right)$ was estimated as the mean diameter of each hexagonal cell composing the foam solid pattern. The thickness of the filaments (i.e. $d_{p}$ ) was computed as the mean diameter of each filament composing the solid fraction (for more details on the computational methods used to estimate $d_{p}$ from the X-ray tomography see Hildebrand \& Ruegsegger 1997; Schneebeli \& Sokratov 2004).

The foam used in these experiments represents an ideal material for the purposes of this paper: the thin filaments and the open structure of the foam allow us to investigate 

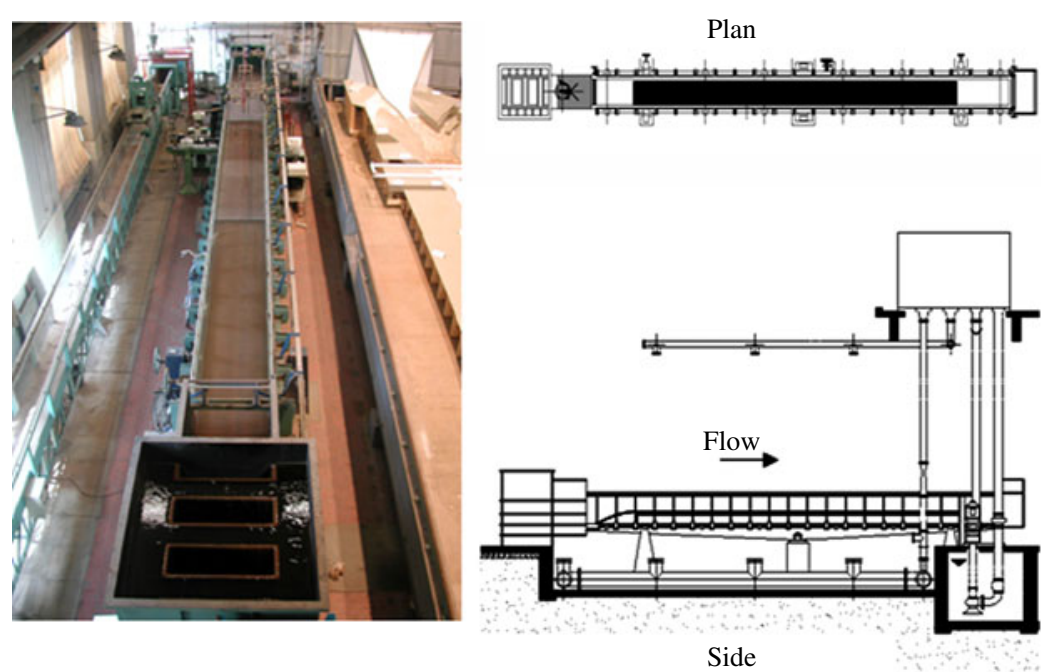

FIGURE 2. (Colour online available at journals.cambridge.org/flm) The open channel flow facility used for the experiments.

$\begin{array}{lccccc}\text { Foam type } & K \times 10^{-9}\left(\mathrm{~m}^{2}\right) & \phi(\%) & d_{p}(\mathrm{~mm}) & \mathrm{SSA}\left(\mathrm{mm}^{-1}\right) & l_{\text {pore }}(\mathrm{mm}) \\ 60 \mathrm{ppi} & 6 & 98.0 & 0.03 & 1.70 & 0.5 \\ 30 \mathrm{ppi} & 39 & 97.0 & 0.11 & 0.82 & 1.5 \\ 10 \mathrm{ppi} & 160 & 96.4 & 0.27 & 0.37 & 3.9\end{array}$

TABLE 1. Properties of the polyurethane foam; the first column identifies the name of the foam as well as its number of pores per inch (ppi); $K=$ permeability; $\phi=$ porosity; $d_{p}=$ filament thickness; SSA $=$ specific surface area, defined as the surface area per unit volume of the solid fraction; $l_{\text {pore }}=$ mean pore size.

flows with relatively small $R e_{d}$ and large $R e_{K}$. Furthermore, the X-ray tomography revealed that polyurethane foam has a homogeneous and isotropic structure, which implies that describing wall permeability by means of a scalar rather than a tensor is a correct assumption.

\subsection{Experimental procedure}

Experiments were carried out in a non-tilting, recirculating, hydraulic flume at the 'G. Bidone' Laboratory of Hydraulics, DITIC, of the Politecnico di Torino (figure 2). The flume is characterized by a straight, glass-sided, rectangular section, which is $18.0 \mathrm{~m}$ long, $0.9 \mathrm{~m}$ wide and $1.0 \mathrm{~m}$ deep. The flow depth at the downstream end of the flume is controlled by means of an adjustable rectangular weir. Beyond this control the water spills freely into a drainage system and is then pumped to a large holding tank placed $8 \mathrm{~m}$ above. Here water is conveyed to the header tank of the flume by means of a pipe where an electromagnetic flow meter is mounted to measure the water discharge. At the upstream end of the flume a series of wire screens were deployed to damp any residual turbulence coming from the header tank.

In order to perform near-wall measurements with the LDA, the bottom of the flume needed to be raised. This was accomplished by covering the entire length of the 


$\begin{array}{lrrrrlllll}\text { Experiment } & \begin{array}{r}K \times 10^{-9} \\ \left(\mathrm{~m}^{2}\right)\end{array} & R_{b} & R e_{\tau} & R e_{K} & R e_{d} & B / \delta & H(\mathrm{~m}) & \begin{array}{c}(\mathrm{d} H / \mathrm{d} x) \\ \times 10^{-3}\end{array} & \text { Symbol } \\ & & & & & & & & & \\ \text { Smooth } & 0 & 53400 & 2160 & 0 & 0 & 15 & 0.100 & -2.53 & * \\ 60 \mathrm{ppiL} & 6 & 47700 & 2349 & 1.9 & 0.790 & 9.4 & 0.096 & -0.927 & \diamond \\ 60 \mathrm{ppiH} & 6 & 62200 & 3234 & 2.2 & 0.912 & 8.2 & 0.113 & -0.983 & \diamond \\ 30 \mathrm{ppiL} & 39 & 30500 & 1856 & 3.2 & 1.76 & 7.80 & 0.116 & -0.963 & 0 \\ 30 \mathrm{ppiH} & 39 & 91400 & 5840 & 7.9 & 4.40 & 6.16 & 0.146 & -1.990 & \bullet \\ 10 \mathrm{ppiL} & 160 & 25700 & 2142 & 8.40 & 4.0 & 8.82 & 0.102 & -3.30 & \square \\ 10 \mathrm{ppiH} & 160 & 38500 & 3848 & 17.2 & 8.2 & 10.06 & 0.090 & -5.26 & \mathbf{\square}\end{array}$

TABLE 2. Hydrodynamic conditions; $R e_{b}=\delta U_{b} / \nu$, where $U_{b}$ is the mean velocity averaged across the boundary layer thickness $\delta ; R e_{\tau}=\delta u_{*} / \nu ; R e_{K}=\sqrt{K} u_{*} / \nu ; R e_{d}=d_{p} u_{*} / \nu ; B / \delta$ is the ratio between flume width and boundary layer thickness; $\mathrm{d} H / \mathrm{d} x$ is the longitudinal gradient of the flow depth. The minus sign indicates that the flow depth decreases along the mean flow direction.

flume with a layer of $20 \mathrm{~cm}$ thick concrete blocks. The smooth wall was obtained by covering the blocks, with five stainless steel panels each of length $2 \mathrm{~m}$ and thickness $2 \mathrm{~mm}$. Once the smooth experiments were finished, the steel panels were used as the bottom of the flume where the permeable walls made of polyurethane foam mattresses could be deployed. Each permeable wall was composed of five panels of $2 \mathrm{~m}$ length, and $0.1 \mathrm{~m}$ thickness. For each wall condition (smooth or permeable), at the upstream end of the flume, a ramp was mounted to connect smoothly the flume bottom with the top of the wall, in order to avoid abrupt flow transitions. For each experiment, the depth of the flow was measured by means of a vernier caliper along all the length of the flume. This allowed estimation of the longitudinal gradient of the hydraulic head, which is reported for each flow condition in table 2.

The test section for turbulence measurements was located $8 \mathrm{~m}$ downstream of the ramp. Here, streamwise $(u)$ and vertical $(w)$ velocities were measured with a two-component LDA in forward scattering mode (see figure 3). This system is characterized by two pairs of laser beams which, after alignment, are associated with the measurement of $u$ and $w$ respectively (for details about this system see Poggi, Porporato \& Ridolfi 2002). Data collection was performed in burst mode using coincidence filtering. The LDA system was mounted on a three-axis manual traverse which allowed control of the positioning of the LDA apparatus with a precision of $\pm 0.025 \mathrm{~mm}$. For each experiment, velocities were measured at about 30 points along a single vertical placed $30 \mathrm{~cm}$ from the sidewalls. For each point, velocities were measured for $600 \mathrm{~s}$ with a sampling frequency which varied between 150 and $500 \mathrm{~Hz}$. With such long time series the $95 \%$ confidence intervals associated with the velocity statistics discussed in this paper are very small, i.e. always smaller than $0.6 \%, 2 \%$ and $5 \%$ for mean velocity, variance and shear Reynolds stress, respectively (confidence intervals were calculated as in DeGraaff \& Eaton 2001). The confidence interval for shear Reynolds stress is the largest because, in LDA applications, the number of simultaneous measurements of $u$ and $w$ is lower than the number of measurements within a single-component time series, typically just a quarter of it.

Near-wall measurements were performed in line with the methodology developed by Poggi et al. (2002). This consists of creating a slot in the wall (as narrow as possible) to allow the passage of the lower vertical laser beam (figure 3). For each wall, the slot width was roughly $2 \mathrm{~mm}$ wide. The measurement point was slightly shifted by a 

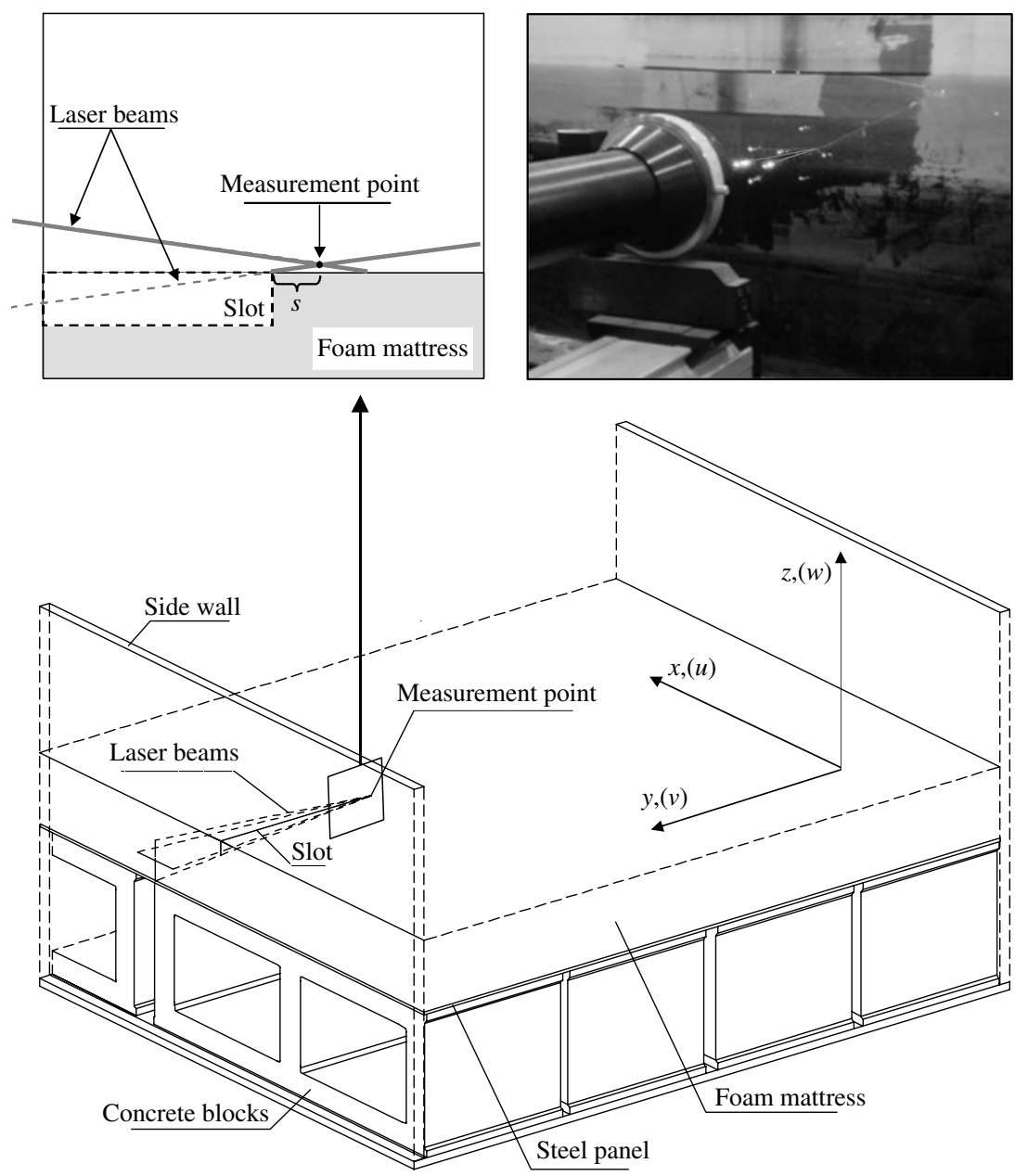

FIGURE 3. Experimental set-up for LDA measurements over permeable walls.

distance $s$ beyond the end of the slot towards the centre of the flume (top left panel of figure 3). This minimized any residual effect of the slot on the flow. Despite this shift, measurements could be performed very close to the wall, i.e. down to $0.4 \mathrm{~mm}$ from the wall surface.

This experimental set-up was validated by Poggi et al. (2002) for the case of smooth walls but not for permeable walls and therefore a permeable wall test was arranged. For one flow and wall condition, streamwise velocity measurements were performed before and after cutting the slot in the foam panels and then velocity statistics were compared. The results (not shown here) showed that the streamwise velocity statistics compared very well between the two cases. This encouraged us to infer that the influence of the slot on the flow was negligible.

Table 2 presents a summary of all the flow conditions used in the experiments. Since the experiments were carried out in a non-tilting flume, uniform flow conditions could not be achieved. Table 2 reports the longitudinal gradient of the flow depths measured for each experiment. Such a non-uniformity may impose a negative mean vertical velocity which can influence the velocity statistics as it occurs in 'suction' turbulent 


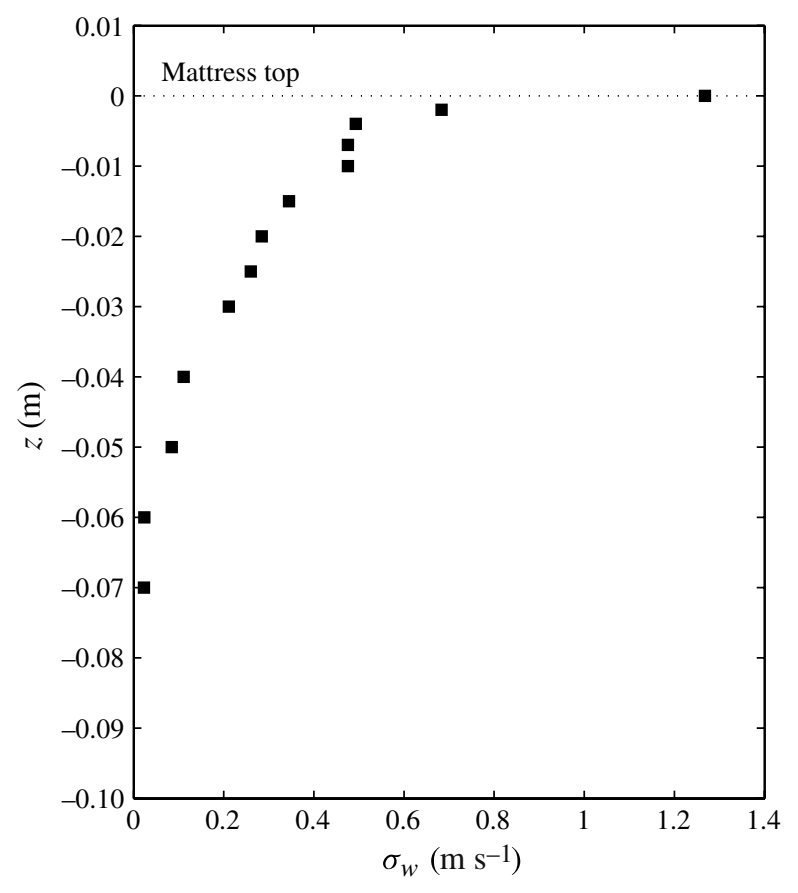

FIGURE 4. Standard deviation of the vertical velocity fluctuations within the foam mattress for experiment $10 \mathrm{ppiH}$.

boundary layers. However, LDA measurements have shown that despite such a nonuniformity, mean vertical velocities were very close to zero, being within the range $(1-3) \times 10^{-3} \mathrm{~m} \mathrm{~s}^{-1}$ at any wall distance. Mean vertical velocities of this magnitude would be expected even in uniform flow conditions due to the effects of secondary currents. We therefore conclude that the influence of non-uniformity on velocity statistics can be neglected. For each permeable wall, two Reynolds numbers were investigated. These were chosen using the following three criteria: (i) the roughness Reynolds numbers are similar to those investigated by Breugem et al. (2006) to allow comparisons; (ii) the ratio between flume width and flow depth could not be lower than 5 to ensure that the mean flow was always two-dimensional (Kironoto \& Graf 1994); (iii) the thickness of the foam panels should be larger than the turbulence penetration depth. The latter condition was tested as follows. First it was assumed that the deepest penetration depth would occur for the most permeable wall at the largest Reynolds number investigated (i.e. experiment $10 \mathrm{ppiH}$ ). Then an opening in the permeable wall was created by moving two neighbouring panels a distance of $4 \mathrm{~mm}$ apart. This opening created enough optical access for the vertical laser beams and the receiver of the LDA system. Fluctuations of the vertical velocity component were then measured at various heights within this gap. Figure 4 shows that the r.m.s. of the vertical velocity fluctuations (i.e. $\sigma_{w}$ ) are close to zero at $0.06 \mathrm{~m}$ below the panel tops. This guarantees that the foam mattresses were thicker than the turbulence penetration depth for all the wall and flow conditions used in the experiments presented herein.

The material properties and hydrodynamic conditions chosen for the experiments allowed the effects of permeability on wall turbulence to be fairly well isolated. However, for the sake of a correct interpretation of the results we make the following 
points. (i) The effects of roughness are negligible when comparing results on velocity statistics between permeable walls, but the comparison with the smooth wall is not as straightforward. In fact, although the filament diameter of the permeable walls is small, over the range of bulk Reynolds numbers used in this study, it is sufficiently large to impose a flow resistance, which differs substantially from that for the smooth wall. Therefore a portion of the difference between the smooth wall and the permeable walls could be due to roughness. (ii) Consistently with previous studies, $\sqrt{K}$ is here chosen as the characteristic length scale quantifying the 'penetrability' of the permeable walls. Although this is a commonly used assumption, here we add a word of caution for it. In fact, permeability is a length scale that is associated with laminar flows in porous media, yet it is used as a characteristic length scale of permeable walls under turbulent flows. In general, it is plausible that other parameters directly related to the geometry of the permeable walls (e.g. the specific surface area or the mean pore dimension) may represent a more physically meaningful alternative. However, for the sake of the interpretation of the results presented in this paper, this does not represent a problem because $\sqrt{K}$ is linearly related to all the other parameters reported in table 1 and therefore the choice of one or the other parameter does not influence the interpretation of the results.

\subsection{Estimation of the friction velocity and the boundary layer thickness}

The friction velocity $u_{*}$ is defined as $u_{*}=\sqrt{\tau_{0} / \rho}$, where $\tau_{0}$ is the shear stress acting at the wall surface and $\rho$ is the fluid density. All the experiments were performed in a flat hydraulic flume which therefore does not allow uniform flow conditions. This makes it difficult to estimate accurately the friction velocity because the shear stress acting at the wall surface cannot be assessed from the bulk momentum balance as in pipe, channel or uniform open channel flows. The only alternative is to estimate $\tau_{0}$ from direct measurements of the Reynolds shear stress $\overline{u^{\prime} w^{\prime}}$, where an overbar indicates time averaging and $u^{\prime}, w^{\prime}$ are the zero-mean streamwise and vertical velocity fluctuations respectively. In this study, the wall shear stress $\tau_{0}$ was chosen as $\tau_{0}=-\rho \overline{u^{\prime} w^{\prime}}{ }_{M}$, where $-\overline{u^{\prime} w^{\prime}}{ }_{M}$ is the maximum value of the shear Reynolds stress profile. For the smooth wall case this value was found to be $6 \%$ smaller than the one obtained from the fitting of the log law of the wall to the mean velocity profile by assuming $\kappa=0.38$. This disagreement provides an indication of the uncertainty characterizing the estimation of the friction velocity in these experiments.

In all the permeable-wall experiments, the boundary layer thickness was assumed to be equal to the flow depth measured from the wall to the free surface. This is justified by the fact that the mean velocity profile was observed to be always monotonically increasing for the whole flow depth and the Reynolds shear stress $\overline{u^{\prime} w^{\prime}}{ }_{M}$ was always non-zero and negative at all the measured points. In contrast, for the smooth wall, the boundary layer thickness and flow depth were not coincident. Indeed it was observed that the shear Reynolds stress crossed the zero-value at a level which was well below the water free surface and this was taken as the top of the boundary layer.

\section{Results}

\subsection{Mean velocity profiles and flow resistance}

In the previous literature, considerable effort has been dedicated to investigating whether the log law of the wall is applicable to describe the mean velocity profile 
of turbulent flows over permeable walls. The log law of the wall may be expressed as:

$$
\frac{U}{u_{*}}=\frac{1}{\kappa} \log \frac{z+d}{z_{0}},
$$

where $z$ is the vertical coordinate whose origin is here taken coincident with the top of the foam mattresses or the solid surface in the case of the smooth-wall experiment, $U$ is the time-averaged velocity, $z_{0}$ is the aerodynamic roughness length, $d$ is the zero plane position and $\kappa$ is the von Kármán coefficient, usually considered to be between 0.38 and 0.41 . The zero plane position can be physically interpreted as either the level where momentum is extracted (Jackson 1981) or, alternatively, the level of an effective plane at which attached eddies are found, so that their vertical size scales with $(z+d)$ (Poggi et al. 2004).

The log law of the wall is valid within the so-called overlap layer, which is a flow region where the vertical coordinate $z$ is the only relevant length scale influencing the flow. This layer appears only at high Reynolds numbers and if there is enough scale separation between outer and inner length scales. Within the overlap layer TKE production and dissipation are commonly assumed to be in equilibrium (Townsend 1976). In our experiments we make use of this property to test whether a genuine overlap layer occurs or not. However, since a direct and accurate estimation of the dissipation rate requires sampling frequencies of velocities much higher than those provided by our experimental set-up, the balance between these two terms is checked using an indirect method that is described as following. We have calculated the skewness of the vertical velocity component $\left(S k_{W}\right)$ that, although limited to the contribution of the vertical velocity component, is a measure of the vertical turbulent transport term in the TKE budget. Assuming that the convective transport due to flow non-uniformity is negligible, TKE production and dissipation are likely to be in equilibrium in flow regions where $S k_{W}$ is nearly constant, i.e. in flow regions where there is no net gain or loss of TKE due to turbulent transport (Nikora \& Goring 2000). Figure 5 shows that a clear plateau of $S k_{W}$ occurs at $0.03<z / \delta<0.15$ for all the experiments except for those related to the wall with the highest permeability (empty and filled squares). This suggests that, for these experiments, there is not enough scale separation between inner and outer length scales to allow a genuine logarithmic layer to develop. Interestingly, the outer limit of the plateau in $S k_{W}$ for the other wall conditions is $z / \delta=0.15$ which is commonly considered as the outer limit of the overlap layer in wall bounded flows. This result is encouraging as it provides confidence that the method based on the vertical profile of $S k W$ is effective in testing the presence of an overlap logarithmic layer.

The log law of the wall was fitted to the mean velocity profiles to estimate, $d, z_{0}$ and $\kappa$ within the range identified by the plateau in the vertical profiles of $S k W$. For completeness, the fitting procedure was also applied within the region $0.03<z / \delta<0.15$ to the experiments with highest wall permeability, although a clear overlap layer does not seem to exist.

The fitting procedure was analogous to the one presented in Breugem et al. (2006) and Suga et al. (2010). First, the zero plane position was estimated as the value of $d$ that provided the best fit between the experimental data and a general logarithmic profile. In other words, we assumed that mean velocities are distributed as $U=a x+b$, where $x=\ln (z+d), a=u_{*} / \kappa$ and $b=-\left(u_{*} / \kappa\right) \ln z_{0}$. The chosen value for $d$ is the one which minimizes the residuals between experimental data and $a x+b$. Once $d$ was estimated, the Von Kármán coefficient and the aerodynamic roughness length were computed as $\kappa=u_{*} / a$ and $z_{0}=\exp \left(-b \kappa / u_{*}\right)$ respectively. 


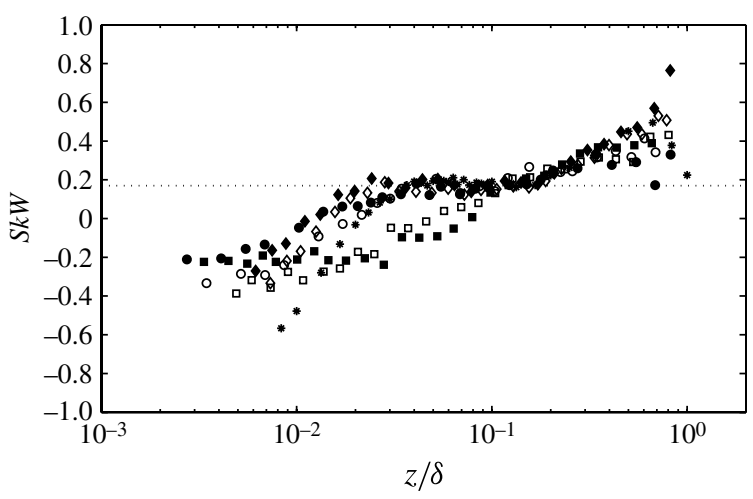

FIGURE 5. Skewness of vertical velocity fluctuations. For the meaning of the symbols the legend is given in table 2 .

$\begin{array}{lccc}\text { Experiment } & \kappa & d(\mathrm{~mm}) & z_{0}(\mathrm{~mm}) \\ \text { smooth } & 0.35 & -0.1 & 0.005 \\ 60 \mathrm{ppiL} & 0.31 & 0.3 & 0.070 \\ 60 \mathrm{ppiH} & 0.32 & 0.3 & 0.090 \\ 30 \mathrm{ppiL} & 0.33 & 0.9 & 0.200 \\ 30 \mathrm{ppiH} & 0.32 & 2.0 & 0.400 \\ 10 \mathrm{ppiL} & 0.33 & 3.5 & 1.100 \\ 10 \mathrm{ppiH} & 0.31 & 5.2 & 2.000\end{array}$

TABLE 3. Results for from the fitting of the log law of the wall to the mean velocity profiles: $\kappa$ is the von Kármán coefficient; $d$ is the zero plane position, and negative and positive values of $d$ indicate that the zero plane position is above and below the wall surface respectively; $z_{0}$ is the roughness length.

The procedure adopted by Breugem et al. (2006) and Suga et al. (2010) is slightly different because they evaluated the zero plane position as the value of $d$ that forced the profile of $(z+d)(\partial U(z) / \partial z)$ to be constant. However, this is equivalent to forcing the linearity of the mean velocity profile in semi-logarithmic coordinates. The results from the fitting procedure are reported in table 3 .

We acknowledge that our procedure may suffer from over-fitting because three parameters are obtained from a linear profile. However, we have adopted it for two reasons: (i) it is consistent with the procedure adopted by Breugem et al. (2006) and Suga et al. (2010) and hence allows comparisons; (ii) the results are similar to those that would be obtained from a two-parameters fitting procedure, i.e. assuming a fixed value of $\kappa$. Indeed, since the values of $\kappa$ obtained with our procedure are fairly constant, the trends of $d$ and $z_{0}$ do not deviate significantly from those that would be obtained assuming a constant $\kappa$. Therefore, we conclude that over-fitting is not a problem concerning the interpretation of our data.

Mean velocity profiles in inner scaling are reported in figure 6. Inner scaling involves normalization of the vertical coordinate with the viscous length scale (figure $6 a$ ) and with wall permeability (figure $6 b$ ). Furthermore, the vertical coordinate origin is corrected with the zero plane position which forces all the mean velocity 

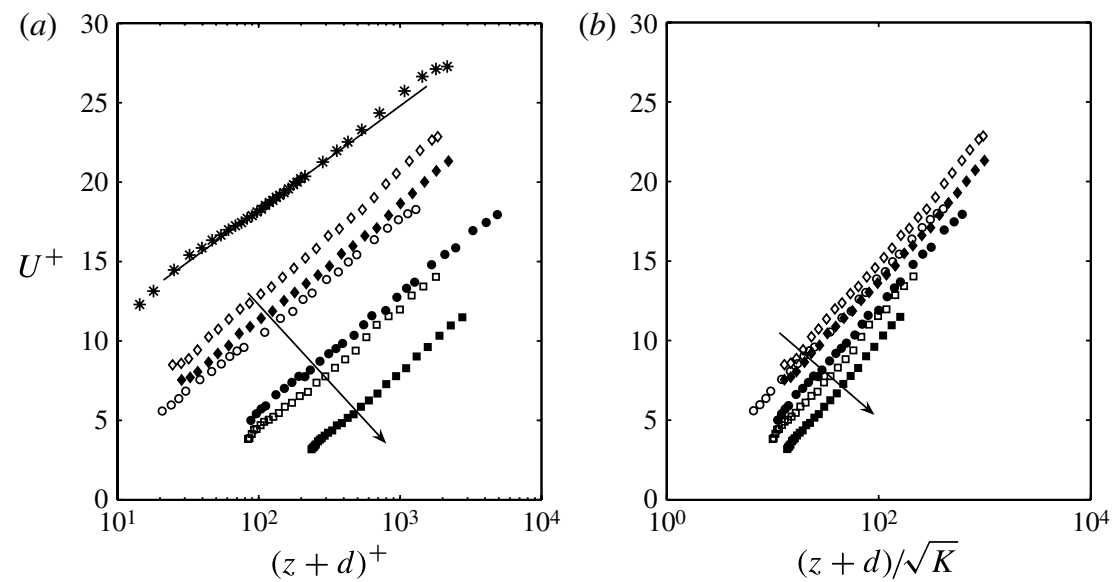

FIGURE 6. (a) Mean velocity profiles with the vertical coordinate scaled in viscous units where the superscript 'plus' indicates normalization with $v / u_{*}$ for lengths and $u_{*}$ for velocities; $(b)$ Mean velocity profiles with the vertical coordinate scaled with the wall permeability. The permeability Reynolds number increases in the direction of the arrows. Legend as in table 2.

profiles to follow a logarithmic behaviour at heights corresponding to $0.03<z / \delta<$ 0.15 . Figure 6 shows that neither of the two scalings makes all the mean velocity profiles collapse into a single curve. However figure $6(b)$ also shows that collapsing of data can be achieved for profiles measured at similar $R e_{K}$. This suggests that flow resistance (i.e. $z_{0}$ ) is a function of $R e_{K}$. The zero plane position, the von Kármán coefficient and the roughness length obtained from the fitting of the mean velocity profiles are all plotted in figure 7 together with the data from Breugem et al. (2006) and Suga et al. (2010). Suga et al. (2010) suggest that in turbulent flows over permeable walls, $\kappa, d$ and $z_{0}$ are a function of $R e_{K}$ and proposed the following empirical relationships:

$$
\begin{gathered}
\kappa=0.365 R e_{K}^{-0.19}, \\
d^{+}=15.1 \operatorname{Re}_{K}-13.5, \\
z_{0}^{+}=6.28 \operatorname{Re}_{K}-9.82,
\end{gathered}
$$

where the plus indicates normalization with the viscous length scale (i.e. $v / u_{*}$ ). Equations (3.3) and (3.4) fit our data reasonably well. However, it should be noted that they do not recover the behaviour of $d$ and $z_{0}$ at the limiting case of $K \rightarrow 0$. At this limiting condition $d$ and $z_{0}$ should tend to values belonging to the turbulent smooth-wall regime. In particular, for $K \rightarrow 0$, then $z_{0} \rightarrow \mathrm{e}^{-\kappa A}$ where $A$ is commonly taken as 5. The behaviour of the zero plane position for smooth walls is more controversial. In most studies it is assumed to be zero but some authors, on the basis of mathematical arguments, have challenged this hypothesis (George \& Castillo 1997; Wosnik, Castillo \& George 2000; George 2007). In particular, Wosnik et al. (2000) argue that, in smooth-wall turbulent flows the zero plane position scales as $d^{+}=-8$, where the minus indicates that the origin is shifted above the wall. On the basis of these considerations we propose the following relationships:

$$
\begin{gathered}
d^{+}=\alpha R e_{K}+\beta, \\
z_{0}^{+}=\gamma \operatorname{Re}_{K}+\mathrm{e}^{-\kappa A},
\end{gathered}
$$


(a)
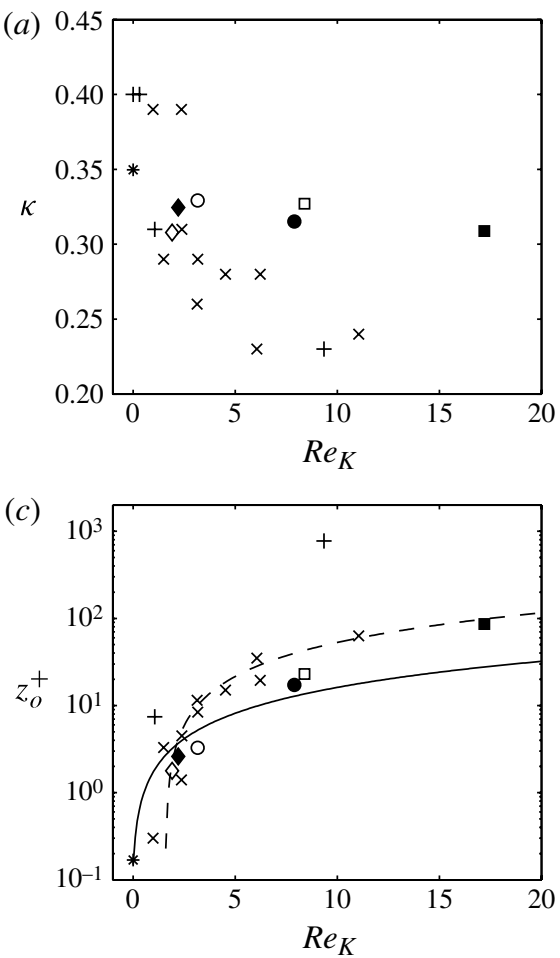
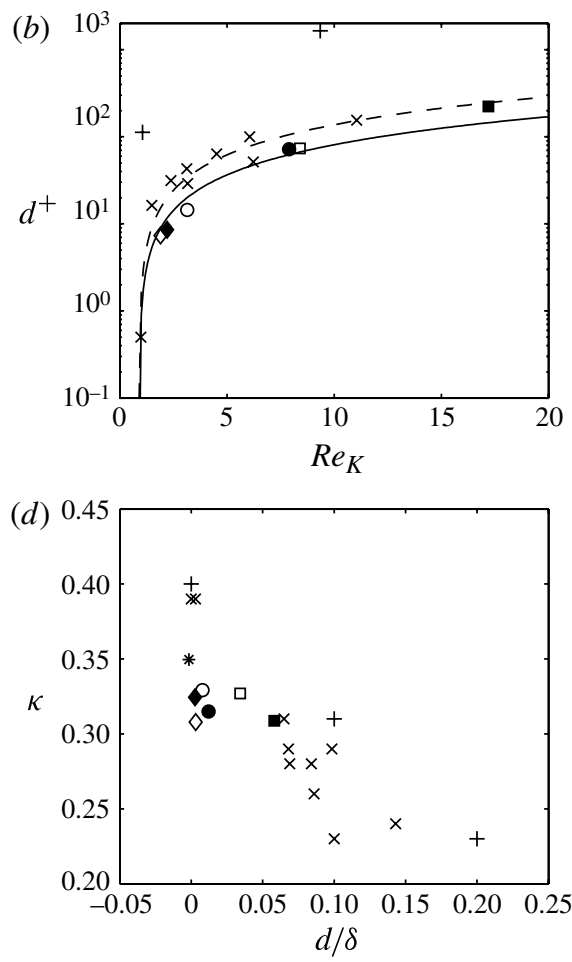

FIgURE 7. (a) Values of the von Kármán constant versus $R e_{K}$; (b) zero plane position in viscous units versus $R e_{K}$, dashed and solid lines indicate equations (3.3) and (3.5) respectively; $(c)$ roughness length in viscous units versus $R e_{K}$, dashed and solid lines indicate equations (3.4) and (3.6) respectively; $(d)$ values of the Von Kármán constant for different $d / \delta$ ratios. For all panels 'plus' and 'cross' signs indicate data from Suga et al. (2010) and Breugem et al. (2006) respectively. The legend for the other symbols is reported in table 2.

where $\beta$ can be either 0 or -8 . The best fit of our data is obtained with $\alpha=8.9$, $\beta=-8$ and $\gamma=1.6$ (see figure $7 b, c$ ). These coefficients were estimated neglecting the data pertaining to the 10 ppi experiments as they did not display a clear logarithmic layer. Equations (3.5)-(3.6) provide a predictive tool to estimate $d$ and $z_{0}$ in turbulent flows over permeable walls with knowledge of wall permeability and the viscous length scale.

Figure 7(a) shows that the von Kármán coefficients derived from our data vary between 0.31 and 0.33 . These values are lower than those commonly reported for smooth or rough walls but are significantly higher than those reported by Breugem et al. (2006) and Suga et al. (2010) for flows over permeable walls at similar $R e_{K}$. This means that the behaviour $\kappa$ is not fully captured by $R e_{K}$ and therefore is not a direct consequence of the relaxation of the wall blocking effect. We suggest that such anomalous values of $\kappa$ are, rather, associated with a poor separation between inner and outer length scales. We argue that, in turbulent flows over permeable walls, the depth of shear penetration and the boundary layer thickness should be taken as the characteristic inner and outer length scales respectively (see also Manes et al. 2011). Building on the work of Jackson (1981), Clifton et al. (2008) argued that in turbulent flows over permeable walls the zero plane position $d$ is a length scale associated with the depth of shear penetration. This implies that, to some extent, $d / \delta$ 


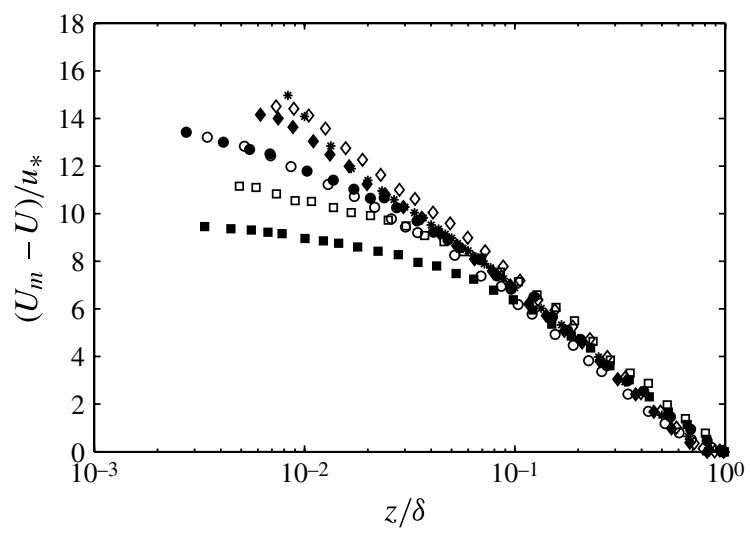

FIGURE 8. Mean velocities in outer scaling. $U_{m}$ is the mean velocity measured at the top of the boundary layer. Legend as in table 2 .

quantifies the ratio between inner and outer length scales. Figure $7(d)$ supports our argument. In fact, when $\kappa$ is plotted versus $d / \delta$ it displays a more consistent trend than in figure $7(a)$. In particular, $\kappa$ decreases with increasing $d / \delta$. Further support for the hypothesis that $\kappa$ depends on the inner-outer scale separation comes from the literature pertaining to rough-wall (impermeable) turbulent boundary layers. Leonardi \& Castro (2010) performed direct numerical simulations of turbulent channel flows over cubical elements with different arrangements. Consistently with our results, they report values of $\kappa$ that consistently decrease with increasing the ratio between an effective roughness (i.e. $z_{0}$ or $d$ ) and the channel height.

We acknowledge that the zero plane position $d$ is a parameter that is just related to the depth of shear penetration and its estimation can be subjected to errors when a logarithmic layer is not fully developed. Therefore, for further experimental studies on this topic, we recommend performing direct estimation of the shear penetration depth although this requires carrying out detailed measurements of turbulent velocity fluctuations within a porous medium, which is a difficult task.

\subsection{On the outer-layer similarity hypothesis}

Outer-layer similarity was tested on first- and second-order velocity statistics. Figures 8 and 9 show that, for $z / \delta>0.4$, mean velocity and Reynolds stress profiles collapse fairly well under the classic outer scaling with normalization by $u_{*}$ and $\delta$.

In contrast with Breugem et al. (2006), our results support the validity of the outer-layer similarity hypothesis for permeable walls. This suggests that departures from similarity in their simulations may be attributed to a low Reynolds number effect. More precisely we argue that, as for the values of the von Kármán coefficients, the results of Breugem et al. (2006) were probably due to a poor separation between inner and outer length scales. An analogous argument was first used by Jimenez (2004) to warn about the validity of Townsend's hypothesis in shallow flows over rough walls. Jimenez (2004) pointed out that outer-layer similarity fails if the ratio between boundary layer thickness and roughness size is too small. We argue that the same principle applies to permeable walls, with the difference that for permeable walls, the effective roughness size is not related to the geometrical size of the roughness elements but to the depth of shear penetration. As discussed in the previous section, in our experiments the ratio between shear penetration depth and boundary layer 

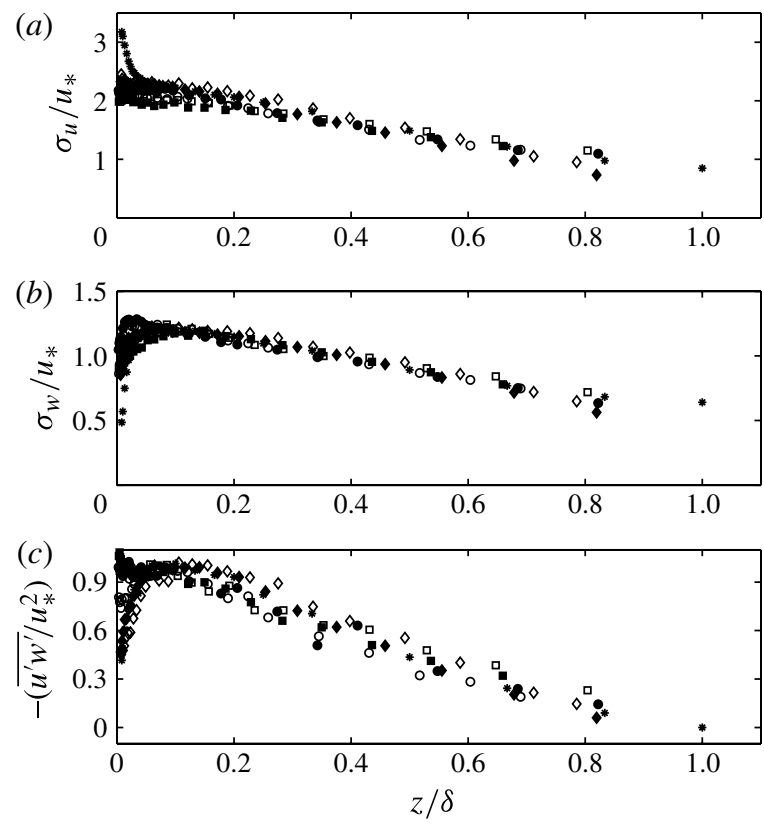

FIGURE 9. (a) Normalized r.m.s. of the streamwise velocity fluctuations; $(b)$ normalized r.m.s. of the vertical velocity fluctuations; $(c)$ normalized Reynolds shear stress. Legend as in table 2 .

thickness is significantly higher than in the simulations of Breugem et al. (2006) (figure $7 d$ ). This provides a plausible reason to explain why our data follow outer-layer similarity and those of Breugem et al. (2006) do not.

\subsection{Turbulence intensities and quadrant analysis}

In figure 10 the standard deviation of the streamwise $\left(\sigma_{u}\right)$ and vertical $\left(\sigma_{w}\right)$ velocity components are plotted in both inner and outer scaling. $\sigma_{u} / u_{*}$ over the permeable walls is significantly smaller than $\sigma_{u} / u_{*}$ over the smooth wall whereas $\sigma_{w} / u_{*}$ is significantly larger. The low values of $\sigma_{u} / u_{*}$ over the permeable walls are probably associated with the absence of the near-wall streaks that instead populate the buffer region of turbulent flows over smooth walls and that are known to contribute significantly to the streamwise turbulence intensity. The high values of $\sigma_{w} / u_{*}$ are instead due to wall permeability, which allows the flow to penetrate within the wall and hence to develop large vertical velocity fluctuations. Conversely, over the smooth wall, the wall blocking condition forces the fluid to move parallel to the wall and hence contributes to damping vertical motions.

For each wall condition, turbulence intensities show a limited dependence on Reynolds number $R e$. Instead $\sigma_{u} / u_{*}$ and $\sigma_{w} / u_{*}$ seem to vary significantly with wall permeability. In the near-wall region $\sigma_{u} / u_{*}$ decreases with increasing $K$. In contrast, $\sigma_{w} / u_{*}$ does not show a similar monotonic behaviour. This is better depicted by figure 11, which reports values of $\sigma_{u, w} / u_{*}$ averaged within a small portion of the near-wall region (i.e. at $0<(z+d) / \sqrt{K}<20)$ for all wall and flow conditions. This figure confirms that, on average, the higher wall permeability is the lower $\sigma_{u} / u_{*}$ is, whereas $\sigma_{w} / u_{*}$ displays an apparent maximum at $K=39 \times 10^{-9}$. This is somewhat 

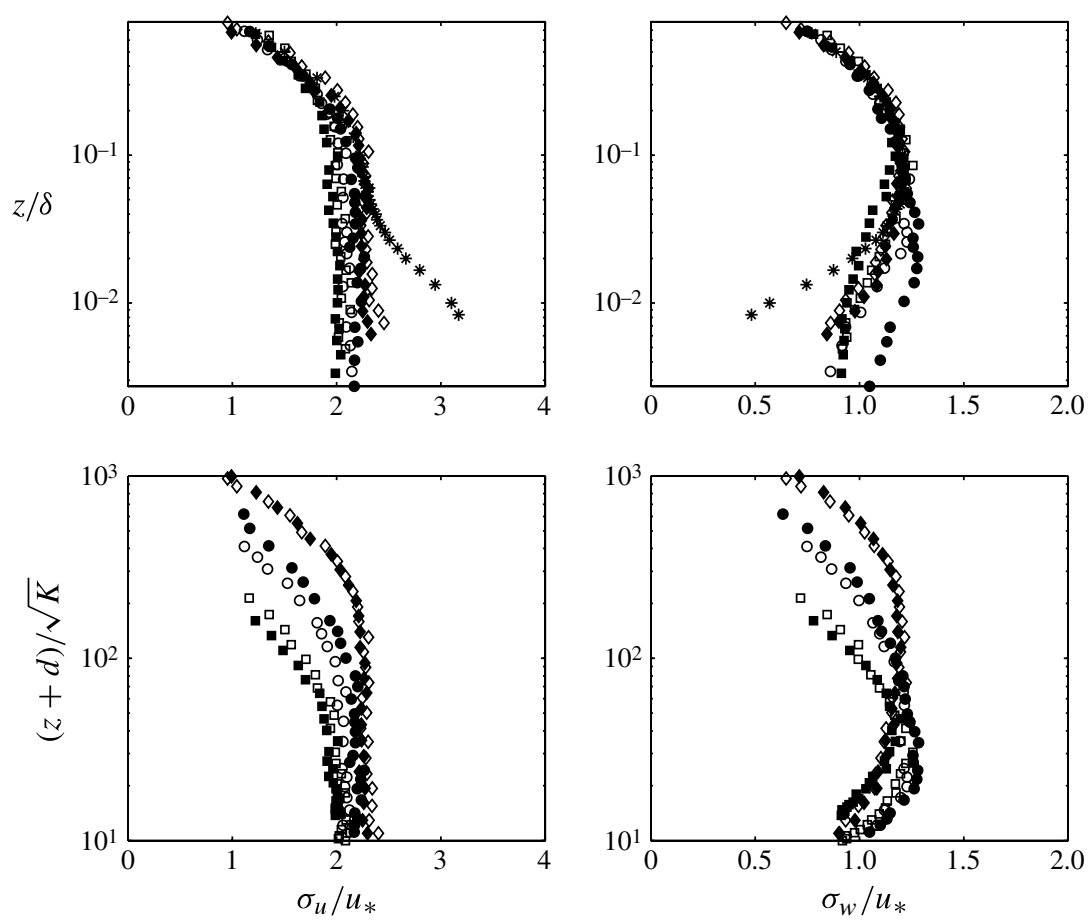

FIGURE 10. Turbulence intensities in inner and outer scaling. Legend as in table 2.

counterintuitive because, as mentioned above, with increasing $K$, the wall blocking condition weakens and one would expect to observe a damping of $\sigma_{u} / u_{*}$ and a consistent increase of $\sigma_{w} / u_{*}$. In other words, as was observed by Suga et al. (2010) and Breugem et al. (2006), one would expect to observe a redistribution of TKE from the streamwise to the vertical velocity component. Our data follow this behaviour only for the walls with low and intermediate permeability whereas for the wall with the highest permeability, $\sigma_{w} / u_{*}$ drops. It should be noted that such a drop in $\sigma_{w} / u_{*}$ is not associated with a drop in $-\overline{u^{\prime} w^{\prime}} / u_{*}$ and therefore the flows over the high-permeability wall are characterized by the highest correlation coefficient between $u^{\prime}$ and $w^{\prime}$ (see figure 12). This suggests that such flows are characterized by the highest degree of coherence and are the most efficient at transporting momentum.

We now make use of quadrant analysis to further investigate how the structure of near-wall turbulence changes with varying wall permeability and to explain the behaviour of turbulence intensities. The quadrant analysis technique identifies flow events associated with the distribution of velocity fluctuations in the four quadrants of the Cartesian plane defined by $u^{\prime}$ (abscissa) and $w^{\prime}$ (ordinate). As such, quadrant 1 $\left(u^{\prime}>0\right.$ and $\left.w^{\prime}>0\right)$, quadrant $2\left(u^{\prime}<0\right.$ and $\left.w^{\prime}>0\right)$, quadrant $3\left(u^{\prime}<0\right.$ and $\left.w^{\prime}<0\right)$ and quadrant $4\left(u^{\prime}>0\right.$ and $\left.w^{\prime}<0\right)$ are associated with flow events defined as inward interactions, ejections, outward interactions and sweeps, respectively. For the sake of clarity we point out that, although the duration of one event can be several sampling velocity time intervals, in our analysis one event is defined by each sample point in the velocity record. The vertical profiles of the ratio of contributions to the Reynolds stress from ejections $\left(Q_{2}\right)$ and sweeps $\left(Q_{4}\right)$ is reported in figure 13 in both inner and outer scaling. 

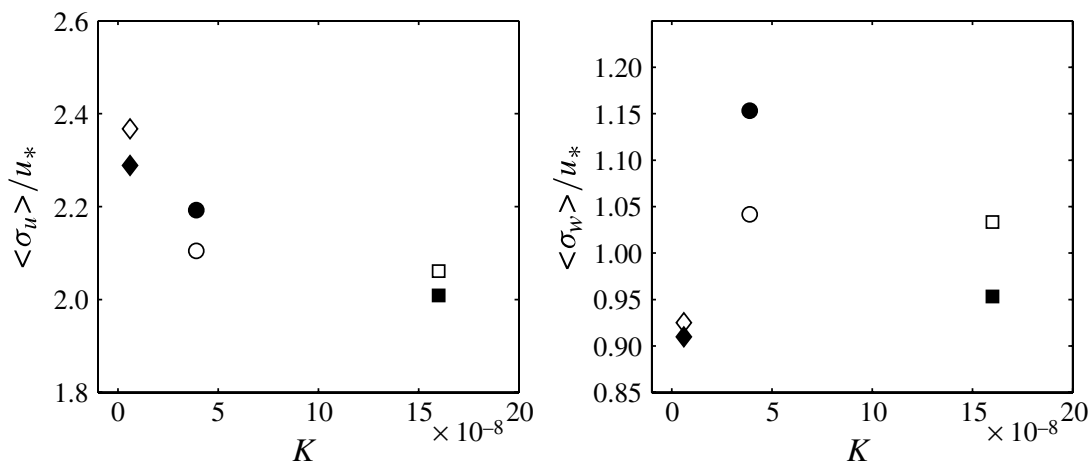

FIGURE 11. Turbulence intensities spatially averaged within the near-wall region, i.e. within the range $0<(z+d / \sqrt{K})<20$. Angle brackets denote the spatial averaging. Legend as in table 2 .
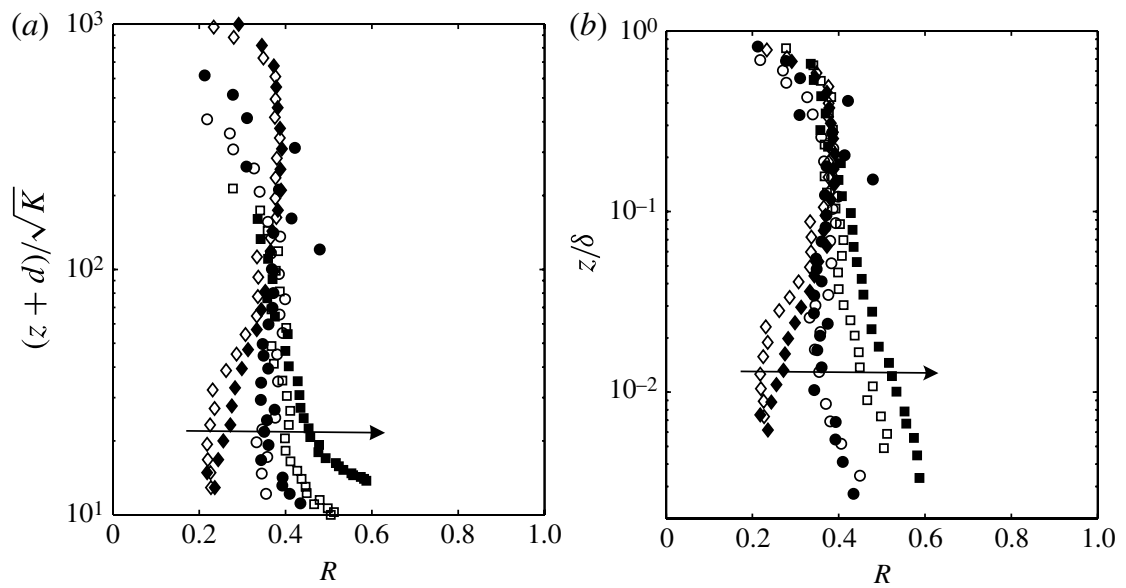

FIGURE 12. Correlation coefficient between streamwise and vertical velocity fluctuations in inner $(a)$ and outer scaling $(b)$. Permeability increases in the direction of the arrows. Legend as in table 2.

Figure 13 shows that for the smooth-wall case, ejections dominate momentum transfer throughout the boundary layer thickness, whereas permeable walls behave similarly to rough walls since they are characterized by a near-wall region where sweeps dominate and an outer region where ejections dominate (Raupach 1981; Katul et al. 2006). Figure 13(b) shows that the crossover between the two regions occurs roughly at $z / \delta=0.1$ for all flow conditions. Furthermore, in the near-wall region, the magnitude of $Q_{2} / Q_{4}$ does not show any obvious dependence on wall permeability.

A more complete picture of quadrant analysis can be obtained not just by looking at the relative magnitude of flow events but also at their relative number. We define $N_{24} / N_{\text {Tot }}$ as the number of ejections and sweeps (i.e. $N_{24}$ ) normalized with the total number of events contained within all quadrants (i.e. $N_{\text {Tot }}$ ). Figure 14 shows that, contrary to $Q_{2} / Q_{4}, N_{24} / N_{T o t}$ does depend on wall permeability within the near-wall region, being higher for higher $K$. This suggests that with increasing $K$ inward and outward interactions are progressively filtered out so that turbulence becomes 

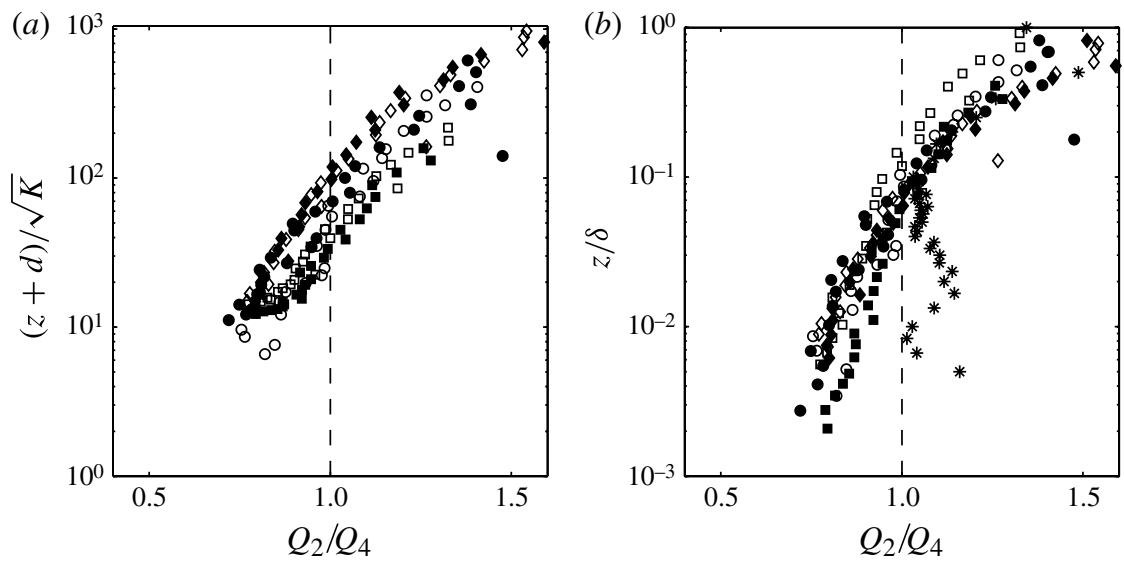

FIGURE 13. Ratio between the contribution of quadrants $2\left(Q_{2}\right)$ and $4\left(Q_{4}\right)$ to the shear Reynolds stress: $(a)$ inner scaling; $(b)$ outer scaling.
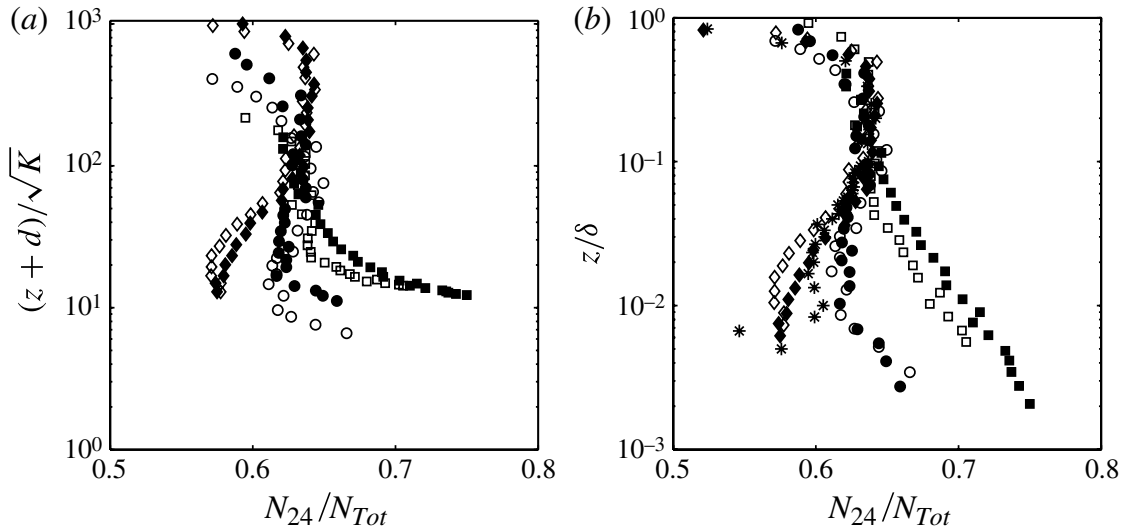

FIGURE 14. Number of events contained within quadrant 2 and 4 (i.e. $N_{24}$ ) normalized with the total number of events contained within all quadrants (i.e. $\left.N_{T o t}\right) ;(a)$ inner scaling; (b) outer scaling. Permeability increases in the direction of the arrows. Legend as in table 2.

more coherent and dominated by a succession of ejections and sweeps. Figure 15 is a visualization of this behaviour directly into quadrant maps and provides further insights. Figure 15 basically compares the joint probability density function (p.d.f.) of $\left(u^{\prime} / u_{*}, w^{\prime} / u_{*}\right)$ measured at $(z+d) / \sqrt{K}=20$ between experiments corresponding to low, intermediate and high wall permeability, respectively. The colour scale indicates the percentage of points contained within each bin of the joint p.d.f. This figure shows that, as wall permeability increases the cloud of points in the quadrant map tilts and shrinks. The shrinking is particularly evident for the high-permeability wall. The tilting of the cloud is associated with an increase of $\sigma_{w} / u_{*}$ and a decrease of $\sigma_{u} / u_{*}$ because the magnitude of vertical velocity fluctuations increases whereas the magnitude of the streamwise velocity fluctuations decreases. In contrast, shrinking is associated with the damping of both components $\sigma_{w} / u_{*}$ and $\sigma_{u} / u_{*}$. This means that with increasing wall permeability $\sigma_{u} / u_{*}$ always decreases whereas for $\sigma_{w} / u_{*}$ there are two counteracting mechanisms that determines its final value. On the one 

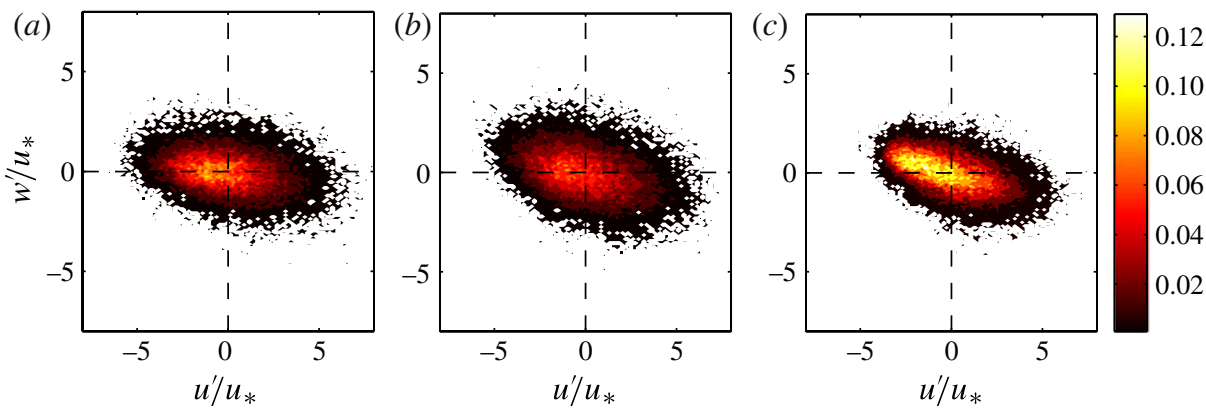

FIgURE 15. (Colour online) Quadrant analysis maps for experiments $60 \mathrm{ppiH}(a)$, $30 \mathrm{ppiH}(b)$ and $10 \mathrm{ppiH}(c)$ at $(z+d) / \sqrt{K}=20$. Wall permeability increases from $(a)$ to $(c)$. Colour-bar indicates the frequency of events contained in each bin of the quadrant map.

hand, by increasing $K$, vertical velocity fluctuations increase because the flow can penetrate better into the wall (tilting). On the other hand an increase in $K$ induces an increase in flow coherency which contributes to damp $\sigma_{w} / u_{*}$ (shrinking). This suggests that, when switching from the intermediate to the highest wall permeability, the second mechanism overcomes the first and therefore $\sigma_{w} / u_{*}$ drops (figure 11). The drop of $\sigma_{w} / u_{*}$ contributes to increasing the correlation coefficient $R$, which for the high-permeability wall is similar to those reported for canopy flows where the mixing layer analogy holds (see e.g. Finnigan 2000). It is therefore plausible that between the intermediate- and the high permeability wall, the flow switches to - mixing layer type of behaviour. In the next section we further substantiate this hypothesis and provide more insights into the scales of turbulence populating the near-wall region at each flow condition investigated.

\subsection{Spectral analysis}

Ghisalberti \& Nepf (2002) point out that in turbulent flows over a fairly dense canopy, the inflection of the mean velocity profile is susceptible to Kelvin Helmoltz (KH) instabilities, which determines shear instability eddies with a characteristic frequency $f_{K H}$ that scales as $f_{K H}=0.032 \bar{U} / \theta$, where $\theta=\int_{-\infty}^{\infty}\left((1 / 4)+((U-\bar{U}) / \Delta U)^{2}\right) \mathrm{d} z$ is the momentum thickness; $\Delta U=U_{m}-U_{1} ; \bar{U}=\left(U_{m}+U_{1}\right) / 2 ; U_{m}$ is the mean streamwise velocity at the top of the boundary layer; $U_{1}$ is the undisturbed velocity within the porous medium, which results from the balance between drag forces and the bulk hydraulic gradient (i.e. $\mathrm{d} H / \mathrm{d} x$ ). Assuming that the drag is mainly due to viscous forces implies the validity of Darcy's law and hence $U_{1}=(\mathrm{d} H / \mathrm{d} x) g K / v$, where $g$ is the gravitational acceleration. The computation of $\theta$ requires knowledge of the mean velocity profile within and above the wall; however, White \& Nepf (2007) point out that, provided that the outer scale of the flow is much larger than the depth of penetration of shear within the wall (this seems to be a valid assumption for the flow and wall conditions considered herein, see figure $7 d$ ) $\theta$ can be estimated reasonably well just from the mean velocity profile above the wall, i.e. $\theta=\int_{0}^{\delta}\left((1 / 4)+((U-\bar{U}) / \Delta U)^{2}\right) \mathrm{d} z$.

If the mixing layer analogy holds, then pre-multiplied power spectra of velocities should display a peak at frequencies $f_{u, w}$ that scale as $f_{u, w} \theta / \bar{U}=0.032$. Figure 16 reports peak frequencies of spectra normalized with the momentum thickness and $\bar{U}$ for all flow conditions and velocity components. In the near-wall region, 

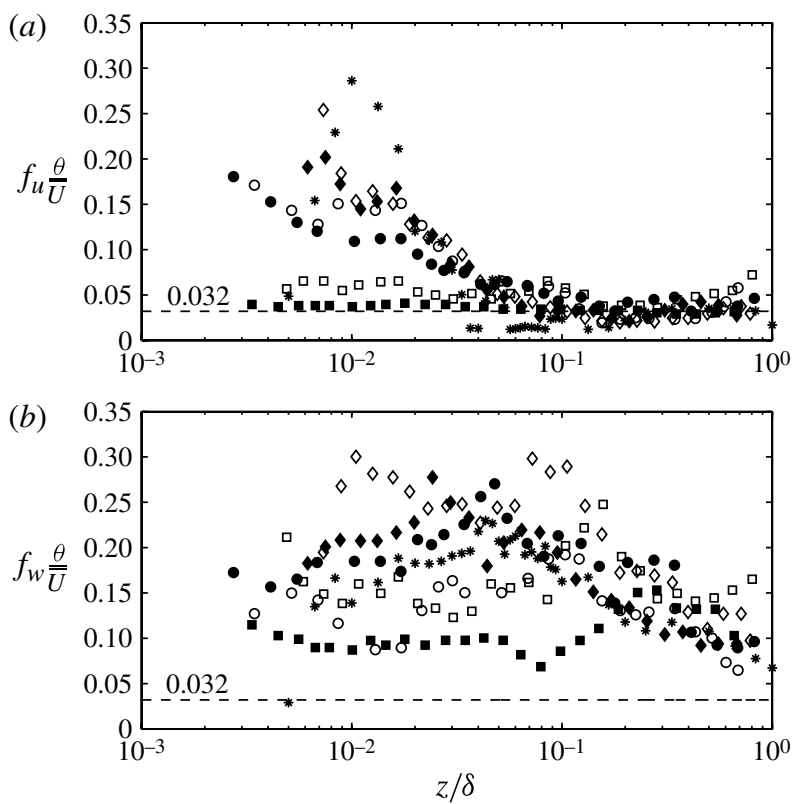

FIGURE 16. Normalized frequencies corresponding to maxima in the pre-multiplied spectra of the streamwise $(a)$ and vertical $(b)$ velocity components. Legend as in table 2.

the normalized peak frequency of the streamwise spectra (i.e. $f_{u}$ ), approach values that are similar to those predicted by the mixing layer analogy only for the wall with the highest permeability. The other wall conditions are characterized by spectral maxima at much higher frequencies. This result substantiates the hypothesis that the flows over the wall with the highest permeability satisfy the mixing layer analogy, whereas the flows over the low and intermediate permeability walls do not. It also suggests that, for these two wall conditions, the inflection point of the mean velocity profile (naturally present at the interface between a free fluid and a permeable wall) is not dynamically active. However, this contrasts with the linear stability analysis of White \& Nepf (2007), who suggest that in flows where the permeable wall is the major sink of momentum (as in the case of the experiments presented herein), the instability of the inflected mean velocity profile requires only the condition of wall penetrability to be initiated, i.e. shear instability eddies, similar those reported for mixing layers, should occur even at very low wall permeability. We suggest that, as White \& Nepf (2007) suspected, this result may not be fully general and may need to be refined since it could be dependent on the characteristics of the permeable walls. More discussion on this issue is provided in $\S 4$.

It should be noted that the peak frequencies of the vertical velocity (i.e. $f_{w}$ ) component do not match the values predicted by the mixing layer analogy for any wall condition, including the one with the highest permeability. This is in contrast with the results obtained for the streamwise velocity component and the results of Ghisalberti \& Nepf (2002) and White \& Nepf (2007) who found a consistent behaviour for both velocity components. However, Finnigan et al. (2009) point out that in Ghisalberti \& Nepf (2002) and White \& Nepf (2007), the mixing layer at the canopy top was effectively two-dimensional due to the tight constraints imposed by the flume walls along the direction of vorticity dictated by the $\mathrm{KH}$ instabilities. In the 

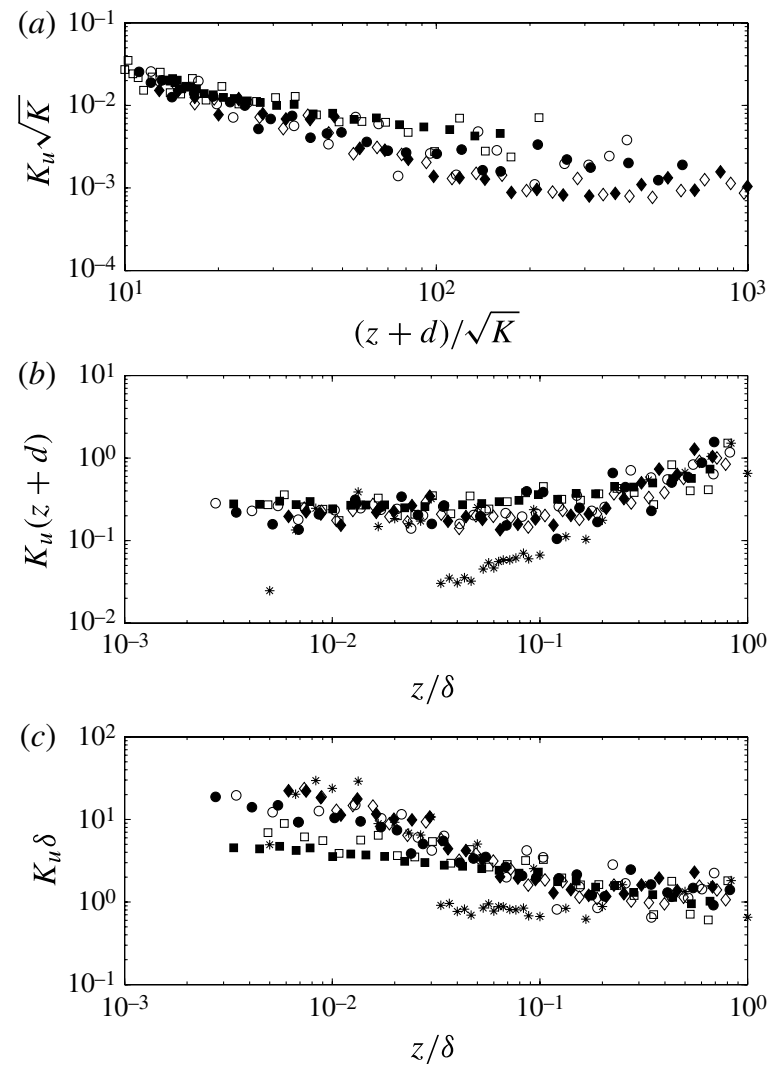

FIGURE 17. Normalized wavenumbers corresponding to maxima in the pre-multiplied spectra of the horizontal velocity component.

case of more three-dimensional mixing layers that are free from these constraints (such as in the case of atmospheric flows over vegetation) Finnigan et al. (2009) observed that, consistently with our results, the imprint of $\mathrm{KH}$ instabilities is best visible in the spectra of the streamwise velocity component. Another interesting feature of figure 16 is that for $z / \delta>0.1$, normalized frequencies for all the wall conditions are in line with those predicted by the mixing layer analogy. While we do not have a full argument to explain this phenomenon, we point out that an analysis of the flow structure based on velocity measurements performed at $z / \delta>0.1$ could be misleading for determining whether a mixing layer analogy holds or not and, to this end, near-wall measurements are strictly necessary since eddies generated from $\mathrm{KH}$ instabilities are bounded to the near-wall region.

A further attempt to characterize the scales of turbulence populating flows over permeable walls is provided by figures 17 and 18. These figures report the spectral peak frequencies of the streamwise and the vertical velocity components respectively. Peak frequencies $f_{u, w}$ were transformed to wavenumbers $K_{u, w}$ by invoking the Taylor hypothesis so that $K_{u, w}(z)=2 \pi\left(f_{u, w} / U(z)\right)$. For each figure, panels $(a)$ report wavenumbers normalized with inner variables such as wall permeability and the zero plane position. In panels $(b)$, wavenumbers are scaled with $(z+d)$ and in panels $(c)$ we report the classical outer-layer scaling using $\delta$ as the characteristic length scale. 

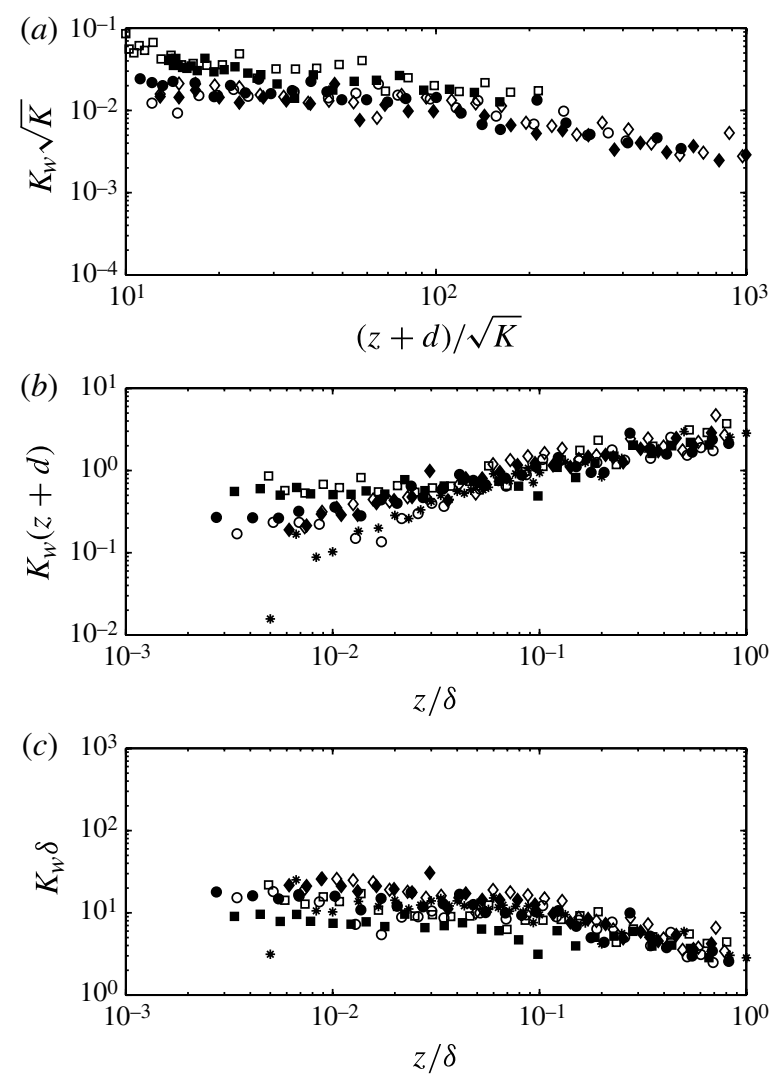

FIGURE 18. Normalized wavenumbers corresponding to maxima of the pre-multiplied spectra of the vertical velocity component.

Interestingly, figure 17(a) shows that $K_{u} \sqrt{K}$ collapses fairly well in the near-wall region for all permeable-wall conditions. This result should be taken with care because at these locations the Taylor hypothesis is not strictly applicable (see e.g. De Alamo \& Jimenez 2009); however it suggests that wall permeability may provide a universal scaling for the longitudinal dimension of near-wall structures over permeable walls, regardless of whether the mixing layer analogy holds or not. The same scaling does not provide an equally good collapse for $K_{w}$ (figure 18a) because the values pertaining to the experiments with the highest wall permeability are significantly higher than all the others, which instead are well grouped at roughly $K_{w} \sqrt{K}=0.02$. Moreover, for the highest wall permeability, the peak wavenumbers of the vertical velocity component are significantly higher than those of the streamwise component, whereas for the other permeable walls they are very similar. This is a further confirmation that the wall with the highest permeability behaves as a canopy with an active mixing layer since in numerical and experimental studies on canopy flows, it is commonly observed that the streamwise velocities are characterized by spectra that peak at wavenumbers that are between a quarter and a half of those of the vertical velocity component (Finnigan et al. 2009).

Concerning the logarithmic layer (i.e. $0.05<z / \delta<0.15$ ), figure 17 shows that for the streamwise velocity component the best collapse of data is provided by the 
outer scaling (figure 17c). Instead, the best collapse of data for the vertical velocity component is obtained when wavenumbers are scaled with the distance from the wall $(z+d)$ (figure 18). These results indicate that, in analogy with turbulent boundary layers over smooth and rough walls, within the log layer, the streamwise velocity component feels the presence of large scales of motion whose size scales with the boundary layer thickness. On the other hand, the vertical velocity component scales with the distance from the wall consistently with the attached eddy model first introduced by Townsend (1976) and then refined by many subsequent works (see e.g. Kunkel \& Marusic 2006; Nickels et al. 2007). This suggests that attached eddies populate the near-wall region of all the flows investigated herein. However, while this is plausible for the smooth wall and the permeable walls with low and intermediate permeability, it is less likely to be true for the wall with the highest permeability. Indeed, it should be noted that the scaling in figure $18(b)$ requires a reliable estimation of $d$, which for this wall condition cannot be obtained due to the lack of a genuine logarithmic layer. Furthermore the lack of such a layer is consistent with the absence of attached eddies (Nikora 2010). Therefore we conclude that the dominant eddy structure for this wall condition is better depicted by figure 16 and hence that shear instability rather than attached eddies dominate the near-wall turbulence structure.

Concerning the outer layer (i.e. $0.4<z / \delta<1$ ), the spectral peaks for both the streamwise and vertical velocity components scale well with the boundary layer thickness (figures $17 c$ and 18c) as also occurs for smooth- and rough-wall boundary layers.

Figure 17(c) shows that within the logarithmic layer, the spectral peaks for the smooth wall occur at much lower wavenumbers than for permeable walls. Peaks for the smooth wall occur at $K_{w} \delta \approx 1$, which corresponds to eddies with a wavelength of about $7 \delta$. In turbulent shear flows, such spectral peaks are usually associated with the occurrence of the so-called very large scale motions (VLSM) (Kim \& Adrian 1999; Guala, Hommema \& Adrian 2006; Monty et al. 2009). The VLSM spectral peak has been observed in the logarithmic layer of smooth and rough canonical shear flows at high Reynolds numbers (Monty et al. 2007; Allen et al. 2007; Monty et al. 2009; Guala, Metzger \& McKeon 2011) and the dynamics of such scales has attracted a lot of attention in the past 15 years because they retain much of the TKE energy of the flow at all positions above the wall and because they are responsible for the failure of classical inner scaling in collapsing turbulence intensities at different Reynolds numbers. To the authors' best knowledge the effect of wall permeability on VLSMs has never been discussed in the literature. In the following we attempt to provide some insights. Figure 19 shows a sample of spectra computed for the streamwise velocity component at $z / \delta=0.05$ (i.e. within the logarithmic layer, when it exists) and plotted in pre-multiplied form. This figure confirms that the smooth wall is characterized by a pronounced peak at $K_{u} \delta \approx 1$ whereas the spectral peak for the permeable walls is shifted at higher wavenumbers, i.e. within the range $K_{u} \delta=2-4$. As already discussed for the von Kármán coefficients and the outer-layer similarity hypothesis, this result may be a shortcoming of a poor separation between inner and outer length scales, which seems to be a fundamental prerequisite to detect VLSMs by means of spectral analysis. Indeed in smooth-wall flows the spectral peak of VLSM is not clearly visible unless $\operatorname{Re}_{\tau}=\delta u_{*} / \nu>1700$ (Hutchins \& Marusic 2007). For smooth walls, $R e_{\tau}$ is the parameter quantifying the separation between inner $\left(\nu / u_{*}\right)$ and outer $(\delta)$ scales. Our experiments well exceed the critical value for $R e_{\tau}$; however, for permeable walls this parameter does not truly quantify the separation between inner and outer scales because the viscous length scale $v / u_{*}$ is not representative for the inner layer. 


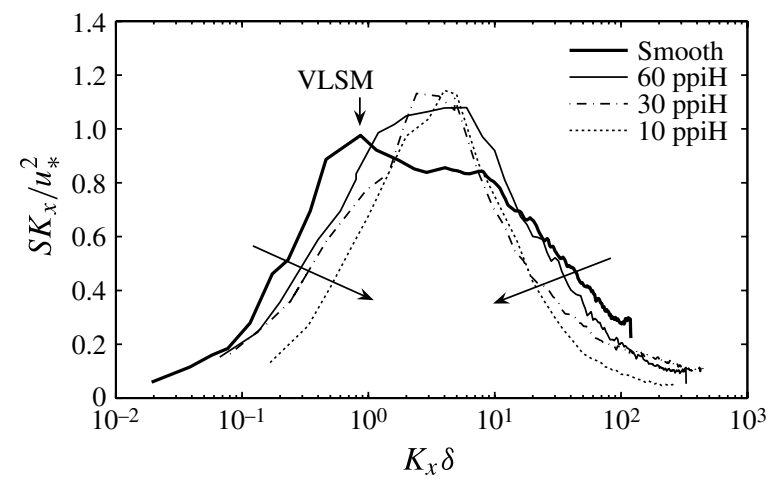

FIGURE 19. Pre-multiplied spectra calculated at $z / \delta=0.05 . K_{x}$ is the wavenumber calculated using the Taylor's hypothesis, i.e $K_{x}=2 \pi f / U$, where $f$ is the frequency and $U$ is the time-averaged velocity measured at $z / \delta=0.05$ for each experiment.

As it was argued in $\S 3.1$, in turbulent flows over permeable walls inner-outer scale separation is, rather, quantified by $d / \delta$. Unfortunately, since no VLSMs were observed in the spectra of any of our experiments, we are not able to estimate the critical value for $d / \delta$ at which VLSM are observable by means of spectral analysis.

Figure 19 shows another interesting feature. As wall permeability increases, the spectra peak at a progressively narrower wavenumber band and hence 'shrink'. From a physical point of view, the more the spectra shrink, the more the flow is dominated by a single scale of motion. Conversely, the flatter the spectrum is the higher the scale heterogeneity of the flow is. A good measure of spectral shrinking and scale heterogeneity is provided by the Shannon entropy of the spectral components (Wesson, Katul \& Siqueira 2003). The spectral Shannon entropy is calculated as

$$
S H=\left(-\sum_{i}^{M} S_{i} \ln S_{i}\right) / \ln (M)
$$

where $S_{i}$ are the spectral density components normalized so that $\sum_{i} S_{i}=1$ and $M$ is the number of spectral estimates. The normalization with $\ln (M)$ ensures that $S H$ is bounded between 0 and 1 and that any difference in $S H$ due to different sampling frequencies is minimized (Wesson et al. 2003). The more the spectral energy is concentrated at one peak the lower is $S H$; conversely, the flatter is the spectrum the more $S H$ tends to 1 , which is the representative value for white noise spectra. Figures 20(a) and 20(b) show the Shannon entropy calculated for the spectra of the streamwise and vertical velocity component respectively. Interestingly, at $z / \delta<0.1$, $S H$ seems to be a function of $R e_{K}$, being lower for higher $R e_{K}$. In particular, $S H$ for the smooth wall and the wall with the highest permeability are significantly different from all the other wall conditions that, overall, are characterized by similar values. Furthermore, for the wall with the highest permeability, $\mathrm{SH}$ approaches the value of 0.6 reported for atmospheric flows over tall vegetation where the mixing layer analogy holds (Wesson et al. 2003).

Going from the smooth to the most permeable wall, there is a transition between a condition where there is a wide spectrum of turbulent eddies to one where the near-wall structure is, rather, influenced by just one scale that, according to the mixing layer analogy, is set by the instability of the mean velocity profile. Interestingly, the Shannon entropy of the smooth wall is significantly larger than those of the permeable 

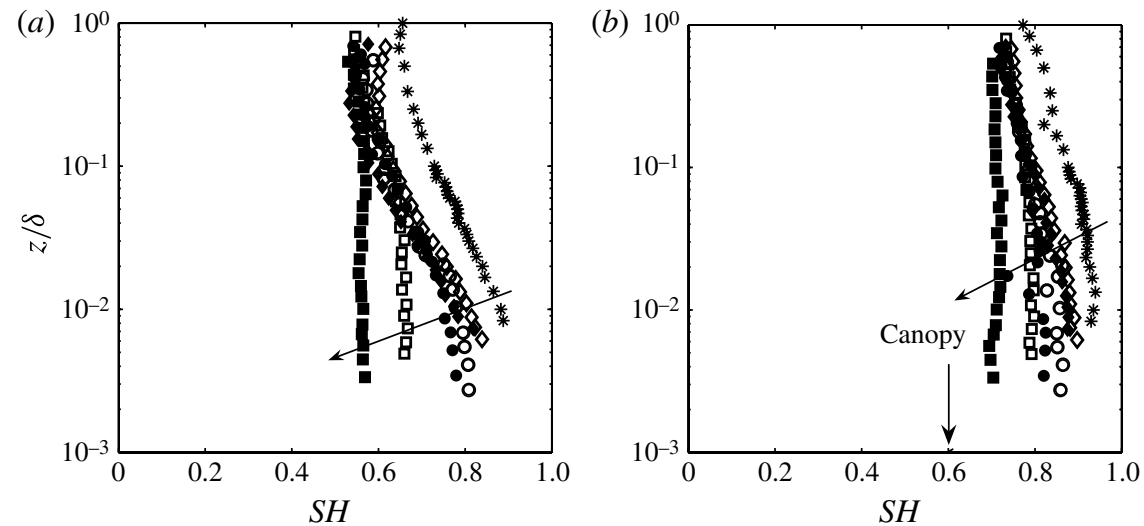

FIGURE 20. Shannon entropy evaluated from spectra of the streamwise $(a)$ and vertical (b) velocity component. $R e_{K}$ increases in the direction of the arrows. Legend as in table 2.

walls throughout the boundary layer thickness and for both velocity components. This could be explained as an effect of VLSMs. Indeed, VLSMs are known to hold a significant fraction of TKE throughout the boundary layer thickness (Marusic et al. 2010) and therefore they can set the largest scales of motion at any height above the wall. Therefore, VLSMs favour the formation of a wide spectrum of scales and hence large values of $S H$ at all positions above the wall.

\section{Discussion and conclusions}

This paper discusses how wall permeability influences the near-wall structure and the scaling of turbulent boundary layers. We have investigated the evolution of the turbulence structure between two limiting conditions represented by a smooth impermeable wall and a very permeable wall which allows the development of a perturbed mixing layer commonly encountered in canopy flows. It was observed that the flow resistance and the depth of shear penetration increase with increasing $R e_{K}$. We have argued that the depth of shear penetration defines the characteristic inner length scale of turbulent flows over permeable walls and is related to the zero plane position, which can be estimated from the mean velocity profiles above the wall. We have shown that the anomalous values of the von Kármán coefficient $\kappa$ reported in the previous literature for turbulent flows over permeable walls seem to be an effect of a poor separation between inner and outer length scales (i.e. $d / \delta$ ) and not simply a consequence of the relaxation of the wall blocking due to permeability, as was cautiously argued by Breugem et al. (2006). In line with the arguments developed for rough-wall boundary layers by Jimenez (2004), we have pointed out that a poor separation between inner and outer scales could be also responsible for the deviations from the outer-layer similarity hypothesis observed in the previous literature on turbulent flows over permeable walls.

The structure of flows over permeable walls is characterized by a near-wall flow region where sweeps dominate and a region, away from the wall, dominated by ejections. With increasing wall permeability, outward and inward interactions are progressively filtered out and the near-wall flow becomes more coherent and dominated by a succession of sweeps and ejections. As a result of this the r.m.s. of streamwise velocity fluctuations progressively decreases with increasing permeability 
whereas the r.m.s. of vertical velocity fluctuations follows a non-monotonic behaviour, which is a result of two counteracting mechanisms. On the one hand vertical velocity fluctuations increase with increasing wall permeability because the flow can penetrate better within the wall. On the other hand the increase in wall permeability implies an increase in coherency and hence in the momentum transport efficiency, which implies that to generate the same Reynolds shear stress $\overline{u^{\prime} w^{\prime}}$, smaller velocity fluctuations are required.

We have observed that the wall with the highest permeability allows the development of eddies whose frequency is very close to the most unstable mode of turbulent mixing layers where the inflection of the mean velocity profile triggers KH shear instabilities. Such shear-instability eddies were not detected in the flows over the walls with low and intermediate permeability. We have pointed out that this result is in contrast with the linear stability analysis performed by White \& Nepf (2007) who argued that wall penetrability is the necessary and sufficient condition for the development of such eddies. A possible explanation to reconcile two apparently contradictory results goes as follows: the analysis of the spectral peak frequencies revealed that, except for the wall with the highest permeability, attached eddies populate the near-wall region of all the flows investigated herein (figures 18 and 16). This means that there may be a competing mechanism between shear instability and attached eddies and the former become detectable only if the two eddy types are comparable in size. With increasing wall permeability and consequently shear penetration, shear instability eddies become progressively larger and hence it is plausible that they become significant only for the wall with the highest permeability. This is consistent with the fact that for this wall condition, a genuine logarithmic layer, which occurs when momentum transfer is fully dominated by attached eddies, does not form and the reason can be attributed to the strong influence of shear instability eddies.

On the basis of the results presented in this paper the structure of turbulent flows over permeable walls and its dependence on wall permeability can be summarized as follows. Turbulent flows over smooth impermeable walls (at sufficiently high $R e$ ) are characterized by a well-developed logarithmic layer where momentum transfer is dominated by attached eddies. Below the logarithmic layer the high level of shear of the flow promotes the formation of longitudinal streaks. With increasing permeability, the no-slip condition at the surface is relaxed and hence turbulence can penetrate within the wall. This promotes the disappearance of longitudinal streaks, the development of shear instability eddies and the penetration of attached eddies within the wall. At these conditions the turbulence structure of the near-wall region is dictated by a competing mechanism between shear instability eddies and attached eddies. Attached eddies are known to populate the flow region below the top of the logarithmic layer which is about $0.15 \delta$ deep. Therefore their size must be proportional to $\delta$. The size of shear instability eddies is proportional to the shear penetration depth, which is associated with the zero plane position. When the shear penetration depth is large with respect to the boundary layer thickness, shear instability eddies will dominate the near-wall structure. This may occur for shallow flows over highly permeable walls. In contrast, attached eddies become the dominant mechanism when the boundary layer thickness is much larger than the depth of shear penetration. This can occur for very deep flows over weakly permeable walls.

As a final remark, we point out that the geometry of the permeable media used in this paper is characterized by a very high porosity and small filament thickness and is ideal for investigating the effects of permeability alone on wall turbulence. This makes 
the results of the present study directly comparable with the literature pertaining to aquatic and atmospheric canopy flows (Ghisalberti \& Nepf 2002; Poggi et al. 2004; Finnigan et al. 2009), and also with the DNS of Breugem et al. (2006), but to a lesser extent with flows over permeable granular walls (Manes et al. 2009; Sarkar \& Dey 2010; Detert, Nikora \& Jirka 2010). The reason is that the latter class of porous media is characterized by large roughness elements, which makes the effects of roughness and permeability both significant. Such effects and their interactions should be addressed in further studies.

We kindly thank C. Camporeale and three anonymous reviewers for the discussions on the manuscript and the insightful comments, and Andrew Clifton for providing the data about the foam properties. C. Manes was supported by the Cassa di Risparmio di Torino (CRT) foundation.

\section{REFERENCES}

Allen, J. J., Shockling, M. A., Kunkel, G. J. \& Smits, A. J. 2007 Turbulent flow in smooth and rough pipes. Phil. Trans. R. Soc. Lond. 365, 699-714.

Breugem, W. P., Boersma, B. J. \& UittenbogaArd, R. E. 2006 The influence of wall permeability on turbulent channel flow. J. Fluid Mech. 562, 35-72.

Clifton, A., Manes, C., Rüedi, J. D., Guala, M. \& Lehning, M. 2008 On shear-driven ventilation of snow. Boundary-Layer Meteorol. 126, 249-261.

De Alamo, J. C. \& Jimenez, J. 2009 Estimation of turbulent convection velocities and corrections to Taylor's approximation. J. Fluid Mech. 640, 5-26.

DeGraAfF, D. B. \& Eaton, J. K. 2001 A high-resolution Laser Doppler Anemometer: design, qualification and uncertainty. Exp. Fluids 30, 522-530.

Detert, M., Nikora, V. \& JiRKA, G. H. 2010 Synoptic velocity and pressure fields at the water-sediment interface of streambeds. J. Fluid Mech. 660, 55-86.

Finnigan, J. 2000 Turbulence in plant canopies. Annu. Rev. Fluid Mech. 32, 519-571.

Finnigan, J. J., Shaw, R. H. \& Patton, E. G. 2009 Turbulent structure above a vegetation canopy. J. Fluid Mech. 637, 387-424.

George, W. K. 2007 Is there a universal log law for turbulent wall-bounded flows?. Phil. Trans. R. Soc. Lond 365, 789-806.

George, W. K. \& CAStillo, L. 1997 Zero-pressure-gradient turbulent boundary layer. Appl. Mech. Rev. 50, 11.

Ghis Alberti, M. 2009 Obstructed shear flows: similarities across systems and scales. J. Fluid Mech. 641, 51-61.

GhisAlberti, M. \& NePf, H. M. 2002 Mixing layers and coherent structures in vegetated aquatic flows. J. Geophys. Res. 107 (C2), 3011.

Guala, M., Hommema, S. E. \& AdRian, R. J. 2006 Large-scale and very-large-scale motions in turbulent pipe flow. J. Fluid Mech. 554, 521-542.

Guala, M., Metzger, M. \& McKeon, B. J. 2011 Interactions within the turbulent boundary layer at high Reynolds number. J. Fluid Mech. 666, 573-604.

HAhn, S., JE, J. \& CHOI, H. 2002 Turbulent channel flow with permeable walls. J. Fluid Mech. 450, 259-285.

Hildebrand, T. \& RUegsegger, P. 1997 EA new method for the model-independent assesment of thickness in three-dimensional images. J. Microsc.-Oxford 185 (Part 1), 67-75.

Hunt, J. C. R. \& MorRison, J. F. 2000 Eddy structure in turbulent boundary layers. Eur. J. Mech. B/Fluids 19 (5), 673-694.

Hutchins, N. \& MARUSIC, I. 2007 Large-scale influences in near wall turbulence. Phil. Trans. R. Soc. 365, 647-664.

JACKSON, P. S. 1981 On the displacement height in the logarithmic velocity profile. J. Fluid Mech. 111, 15-25. 
JimeneZ, J. 2004 Turbulent flows over rough walls. Annu. Rev. Fluid Mech. 36, 173-196.

Katul, G., Poggi, D., Cava, D. \& Finnigan, J. 2006 The relative importance of ejections and sweeps to momentum transfer in the atmospheric boundary layer. Boundary-Layer Meteorol. 120, 367-375.

KIM, K. C. \& ADRIAN, R. J. 1999 Very large-scale motion in the outer layer. Phys. Fluids 11, 417-422.

KIRONOTO, B. A. \& GRAF, W. H. 1994 Turbulence characteristics in rough uniform open-channel flow. Proc. Inst. Civil Engrs Waters Maritime Energy 106, 333-334.

Krogstad, P. A., Antonia, R. A. \& Browne, L. W. B. 1992 Comparison between rough-and smooth-wall turbulent boundary layers. J. Fluid Mech. 245, 519-617.

KUnKel, G. J. \& MARUSIC, I. 2006 Study of the near-wall-turbulent region of the high-Reynoldsnumber boundary layer using an atmospheric flow. J. Fluid Mech. 548, 375-402.

LeOnARDi, S. \& CASTRO, I. P. 2010 Channel flow over large cube roughness: a direct numerical simulation study. J. Fluid Mech. 651, 519-539.

Manes, C., Pokrajac, D., McEwan, I. \& Nikora, V. 2009 Turbulence structure of open channel flows over permeable and impermeable beds: a comparative study. Phys. Fluids 21, 125109.

Manes, C., Pokrajac, D., Nikora, V., Ridolfi, L. \& Poggi, D. 2011 Turbulent friction in flows over permeable walls. Geophys. Res. Lett. 38, L03402.

Marusic, I., Mckeon, B. J., Monkevitz, P. A., Nagib, H. M., Smits, A. J. \& Sreenivasan, K. R. 2010 Wall-bounded turbulent flows at high Reynolds numbers: recent advances and key issues. Phys. Fluids 22, 065103.

Monty, J. P., Hutchins, N., NG, H. C. H., Marusic, I. \& Chong, M. S. 2009 A comparison of turbulent pipe, channel and boundary layer flows. J. Fluid Mech. 632, 431-442.

Monty, J. P., Stewart, J. A., Williams, R. C. \& Chong, M. S. 2007 Large-scale features in turbulent pipe and channel flows. J. Fluid Mech. 589, 147-156.

Nickels, T. B., Marusic, I., Hafez, S., Hutchins, N. \& Chong, M. S. 2007 Some predictions of the attached eddy model for a high Reynolds number boundary layer. Phil. Trans. R. Soc. Lond. 365, 807-822.

NikoRA, V. 2010 Hydrodynamics of aquatic ecosystems an interface between ecology, biomechanics and environmental fluid mechanics. River Research and Applications 26, 367-384.

NikoRA, V. \& GoRING, D. 2000 Flow turbulence over fixed and weakly mobile gravel beds. J. Hydraul. Engng 126, 9.

Poggi, D., Porporato, A. \& Ridolfi, L. 2002 An experimental contribution to near-wall measurements by means of a special laser Doppler anemometry technique. Exp. Fluids 32 (3), 366-375.

Poggi, D., Porporato, A., Ridolfi, L., Albertson, J. D. \& Katul, G. G. 2004 The effect of vegetation density on canopy sub-layer turbulence. Boundary-Layer Meteorol. 111, 565-587.

RAUPACH, M. R. 1981 Conditional statistics of Reynolds stress in rough-wall and smooth-wall turbulent boundary layers. J. Fluid Mech. 108, 363-382.

Raupach, M. R., Finnigan, J. J. \& BRunet, Y 1996 Coherent eddies and turbulence in vegetation canopies: the mixing layer analogy. Boundary-Layer Meteorol. 78, 351-382.

SARKAR, S. \& DEY, S. 2010 Double-averaging turbulence characteristics in flows over a gravel bed. J. Hydraul. Res. 48 (6), 801-809.

SchneEbeli, M. \& Sokratov, S. 2004 Tomography of temperature gradient metamorphism of snow and associated changes in heat conductivity. Hydrol. Process. 18, 3655-3665.

Suga, K., Matsumura, Y., Ashitaka, Y., Tominaga, S. \& Kaneda, M. 2010 Effects of wall permeability on turbulence. Intl J. Heat Fluid Flow 31 (6), 974-984.

Townsend, A. A. 1976 The Structure of Turbulent Shear Flow. Cambridge University Press.

Wesson, K. H., Katul, G. G. \& Siqueira, M. 2003 Quantifying organization of atmospheric turbulent motion using nonlinear time series analysis. Boundary-Layer Meteorol. 106 (3), 507-525.

White, B. L. \& NePF, H. M. 2007 Shear instability and coherent structures in shallow flow adjacent to a porous layer. J. Fluid Mech. 593, 1-32. 
Wosnik, M., Castillo, L. \& George, W. K. 2000 A theory for turbulent pipe and channel flows. J. Fluid Mech. 421, 115-145.

ZaGni, A. F. E. \& SMITH, K. V. H. 1976 Channel flow over permeable beds of graded spheres. J. Hydraul. Div, 102 (HY2). 